\title{
Vehicle Technologies towards Sustainable and Energy Efficient Aviation
}

\author{
Jens Friedrichs ${ }^{1}$ \\ Institute of Jet Propulsion and Turbomachinery, Technische Universität Braunschweig, \\ Lower Saxony, 38108 Braunschweig, Germany
}

\begin{abstract}
Ali Elham ${ }^{2}$
Institute of Aircraft Design and Lightweight Structures, Technische Universität Braunschweig, Lower Saxony, 38108 Braunschweig, Germany
\end{abstract}

\author{
Christian Hühne ${ }^{3}$ \\ Institute of Mechanics and Adaptronics, Technische Universität Braunschweig, \\ Lower Saxony, 38108 Braunschweig, Germany \\ Rolf Radespiel $^{4}$, André Bauknecht ${ }^{5}$ \\ Institute of Fluid Mechanics, Technische Universität Braunschweig, \\ Lower Saxony, 38108 Braunschweig, Germany
}

\begin{abstract}
The Cluster of Excellence $\mathrm{SE}^{2} \mathrm{~A}$ - Sustainable and Energy-Efficient Aviation is a joint research initiative of Technische Universität Braunschweig, Leibniz University Hannover, German Aerospace Center, the National Metrology Institute of Germany and the Braunschweig University of Art. The overall mission is research and fundamental technological development for a sustainable future global air transport system. Following the vision of zero fossil fuel usage in aviation in a 2050 timeframe, reducing material and noise emissions while keeping air transport affordable on short to long range missions, $\mathrm{SE}^{2} \mathrm{~A}$ focuses on significantly reducing the aircraft energy demand in combination with future energy storage and conversion technologies. This large design space makes preliminary system design and assessment based on models of different fidelity level indispensable. In this paper, the framework and research structure of the Cluster is described as well as a special focus on relevant and required vehicle technologies. This follows the need for a significant reduction of the mission energy to allow for future alternative propulsion systems and covers means for radical reductions in aircraft drag and dynamic loads, which in turn allow for reduced operating empty weight. An overall aircraft design methodology is employed for integration and assessment of technological progress. Such analysis is used to identify and quantify snowball effects and to prioritize technologies, but also to identify conflicting areas and requirements.
\end{abstract}

\footnotetext{
${ }^{1}$ Professor, Institute of Jet Propulsion and Turbomachinery, Hermann-Blenk-Str. 37.

${ }^{2}$ Professor, Chair of Aircraft Conceptual Design, Hermann-Blenk-Str. 35.

${ }^{3}$ Professor, Institute of Mechanics and Adaptronics, Langer Kamp 6

${ }^{4}$ Professor, Institute of Fluid Mechanics, Hermann-Blenk-Str. 37.

${ }^{5}$ Independent Research Group Leader, $\mathrm{SE}^{2} \mathrm{~A}$ - Sustainable \& Energy Efficient Aviation, Hermann-Blenk-Str. 37.
} 


\section{Abbreviations and Nomenclature}

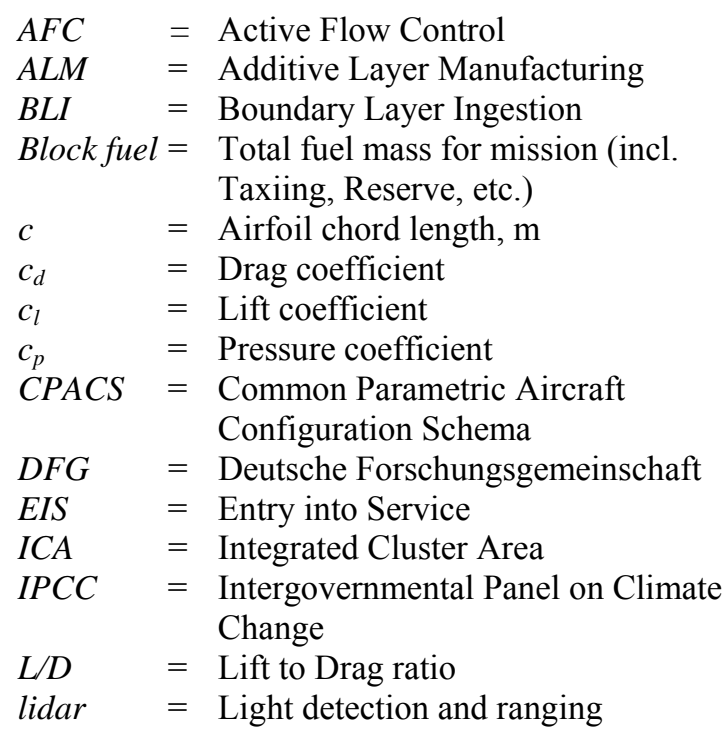

\begin{tabular}{|c|c|}
\hline M & $=$ Mach number \\
\hline MTOW & $=$ Maximum Take-Off Weight \\
\hline PSC & $=$ Power Saving Coefficient \\
\hline$P T L$ & $=$ Power to Liquid \\
\hline$P T X$ & $=$ Power to Gas \\
\hline $\operatorname{Re}$ & $=$ Reynolds number \\
\hline$R U$ & $=$ Research Unit \\
\hline SFC & $=$ (Thrust-) Specific Fuel Consumption \\
\hline$S E^{2} A$ & $\begin{aligned}= & \text { Sustainable and Energy-Efficient } \\
& \text { Aviation }\end{aligned}$ \\
\hline$t$ & $=$ Time, $\mathrm{s}$ \\
\hline$t^{*}$ & $=$ Non-dimensional time \\
\hline TMS & $=$ Thermal Management Systems \\
\hline TPMS & $\begin{aligned} &= \text { Triply Periodic Minimal Surface } \\
& \text { Structures }\end{aligned}$ \\
\hline$U_{\infty}$ & $=$ Freestream velocity, $\mathrm{m} / \mathrm{s}$ \\
\hline & $=$ Spatial coordinate, $\mathrm{m}$ \\
\hline
\end{tabular}

\section{Introduction}

Since 2019 the Cluster of Excellence "Sustainable and Energy-Efficient Aviation - SE $\mathrm{A}^{2}$ " is funded by DFG (Deutsche Forschungsgemeinschaft) and the German Council of Science and Humanities as part of the federal German excellence strategy. $\mathrm{SE}^{2} \mathrm{~A}$ intends to establish the scientific and technological foundations for a sustainable future global air transport system and its required technologies. To meet the global mobility demands in combination with the goals on environmental protection, this translates into drastic reductions of the overall carbon footprint of the air transport system by replacing fossil fuels for air transport operations, as well as into substantially reduced noise emissions, without compromising reliability, safety, and economic viability. To fulfill its mission, the Cluster will especially focus on short, medium and long range aircraft (more than 70 seats), operating solely on renewable energies. The Cluster concentrates on identifying medium- and long-term (year 2050) potential for reducing the carbon footprint of air transport through comprehensive systems-based analysis resulting in new solutions. The institutional members of $\mathrm{SE}^{2} \mathrm{~A}$ are the Technische Universität Braunschweig (leading the Cluster), the Leibniz University Hannover (LUH), the German Aerospace Center (DLR), the National Metrology Institute of Germany (PTB), and the Braunschweig University of Art (HBK). Associated members are the University of Delft (Netherlands) as well as the Airport Hannover. Currently approximately 65 doctoral researchers supervised by 60 PIs are working within research projects and research units of $\mathrm{SE}^{2} \mathrm{~A}$.

\section{Research Framework and Aggregation in $\mathrm{SE}^{2} \mathrm{~A}$}

\section{A. Background and general framework of the Cluster}

Although the Covid-19 crisis has led to a significant drop in global air travel, it is expected that the megatrend "Mobility" will generate significant demands for air transport capacities in the near future. In 2015, transportation alone accounted for $23 \%$ of the worldwide, anthropogenic $\mathrm{CO}_{2}$ emissions, with aviation being responsible for $12 \%$ of the transport-related $\mathrm{CO}_{2}$ emissions ${ }^{6}$. Even assuming that typical total growth rates in commercial air transport volumes of $4 \%$ to $5 \%$ - as seen during the last decades - based on the pre-Covid-19 level will not be caught up the next years, the challenging targets on environmental protection of at least an $80 \%$ reduction in greenhouse gas emissions by 2050 , as set by the IPCC requires revolutionary changes in aviation. While ground transportation will decrease its carbon footprint through electrification, the very high power and energy densities demanded by aviation do not allow for a direct transfer of such technologies.

Besides the $\mathrm{CO}_{2}$ footprint and other gaseous and aerosol emission also noise emissions represent a serious environmental impact of air traffic, as is evident in the international and European legislation on climate and noise

\footnotetext{
${ }^{6}$ Data from ICCT: The State of Clean Transportation Policy (2015)
} 
protection. The number of airports with individual noise abatement and mitigation strategies has significantly grown during the last decade. In the future, airports carrying significant traffic will have to provide good links to ground transit and short door-to-door travel times for passengers. In addition, adoption of complex new technologies in aviation typically has required time frames of decades due to risk-averse decision-making paradigms, resulting in long product-approval cycles and necessarily long product life-cycles. Hence, there is an urgent need for fundamental research that not only identifies promising technologies but also quantifies their potential taking interdependencies into account in order to solve the eneroy problem of air transport

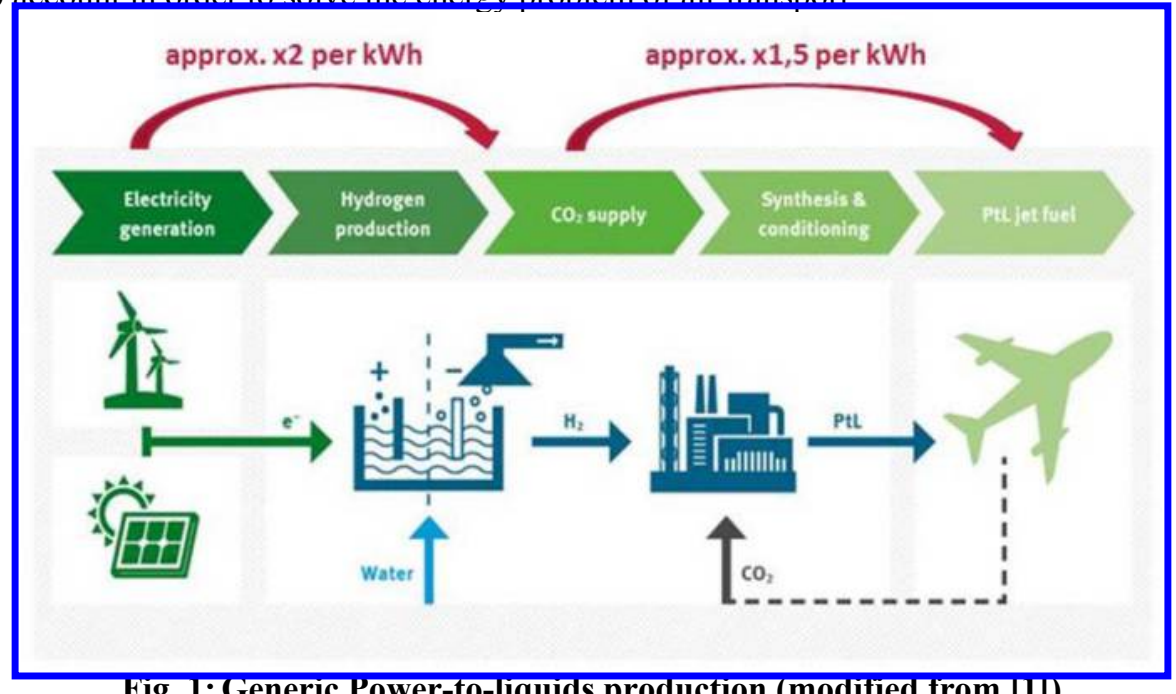

Fig. 1: Generic Power-to-Iiquids production (modified from [I])

As a direct candidate for replacing fossil oil-based liquid fuels with some advantageous properties on energy density, only Power-to-X (PTX; X=gas $(\mathrm{G})$ or liquid (L)) processes are expected to reach the targets for $\mathrm{CO}_{2}$ emissions. Assuming the availability of electric power from renewable resources (wind or photovoltaic energy) at given cost level and an approximated 66\% conversion efficiency, liquid hydrogen is expected to be producible in large-scale processes at a level of roughly a factor of 2 compared to the electric power costs in 2050 (cf. Fig. 1); at a level of $5 \mathrm{US} \$ \mathrm{ct} / \mathrm{kWh}$ renewable electric energy this leads to approx. 8 to $12 \mathrm{US} \$ \mathrm{ct} / \mathrm{kWh}$. For Fischer-Tropsch or methanol-based synthetic liquid fuels synthesis, which offers similar power-to-weight ratios as kerosene, the price increase will be another factor of approximately 1.5 , depending on process efficiency and heat management as well as up-scaling effects. This leads to a price per $\mathrm{kWh}$ of approximately 12 to $20 \mathrm{US} \$ \mathrm{ct}$ for 2050 . However, such SAF fuel might become more competitive if $\mathrm{CO}_{2}$ emission taxes are levied and enforced. $\mathrm{A} \mathrm{CO}_{2}$ emissions tax of e.g. 30 US $\$ c t /$ passenger $/ 100 \mathrm{~km}$ would increase the kerosene fuel cost by approximately $20-25 \%$.

To allow for a careful and balanced assessment of aviation energy conversion, which does not just focus on carbon emission and replacing kerosene by SAF (which covers on its own a wide range of fed stock and processes) but also other solutions like hydrogen and electric-hybrids, a classification into emission-neutral and emissions-free technologies with regard to their gaseous, liquid, and solid emissions is recommended:

Emission-free: No emission that leads to greenhouse impact as well as aerosol effects, contrail or cirrus generation or indirect cloud effects. This can only fully be achieved by battery-based propulsion, which fore sure bears other challenges. Even fuel-cell and renewable $\mathrm{H}_{2}$-based propulsion does not fully support this category since the water emission might contribute to contrail and cirrus generation depending on the thermodynamic properties of the droplets, which is an active research area.

Emission-neutral: Emission during operation equal to capturing of the same components during synthesis process. This applies e.g. to synthetic kerosene based on a PTL process operated with renewable energy. Although being emission-neutral, such technologies do have a greenhouse potential based on the different impact of emissions released in the upper atmosphere compared to its capture on ground. 
Consequently, a change of the energy supply from fossil fuels towards a sustainable system will most probably have to make use of more than just one solution in order to combine economic effects and environmental impact reduction in the most effective way. In other words, electric flying based on hybrid energy storage solutions, even including future batteries, can be such an effective solution by combining lowest energy production cost with best environmental effects, even if this approach is limited to short range and low transport capacity vehicles. On the other hand, liquid hydrogen offers a promising compromise between energy costs and environmental impact, but comes with an unfavorable power-to-volume ratio leading to a storage problem accompanied by heat management challenges using fuel cell based energy conversion. Both challenges can only be addressed by radical new vehicle designs. To cope with the energy demands on semi-long- and long-range missions, no alternative is seen to liquid SAF energy carriers (PTL) - also in a long term run. While the latter most probably requires the smallest changes in vehicle and propulsion technology, it still means a revolution in economies and is thus, with regards to its related costs, strongly coupled to scale-effects in economy and their timeframe, which in turn couples PTL-based aviation as well as $\mathrm{H}_{2}$-based aviation to other sectors of global economy.

Another very important aspect of the economic framework results from typical product life-cycles in aviation, which have been considerably extended during recent decades. Although the Covid-19 crisis currently leads to an early retirement within the fleets of some airlines, the basic mechanism holds true for a wide range of segments and manufacturers. For instance, the Airbus 320 family was officially launched in 1984 and went into service in 1989. Although new members of the family have brought significant improvement in efficiency and reduced operational costs, the basic aircraft has remained the same since and production will most probably continue beyond 2035. Such long life-cycles are a direct consequence of the very high complexity of the entire air transport system and are especially driven by its infrastructure and vehicles. The development of a successful air transport solution primarily depends upon overcoming the challenges posed by the physics of flying. Although the laws governing flight are understood, the creation of airframes offering a simultaneous optimum in functional efficiency and low weight in parallel with an operation for up to 30,000 flight hours is still an engineering challenge. This on its own has driven the development of entirely new materials, design methods, and technologies over the last decades. Overall, new technologies not only have to fulfill the technical and regulatory requirements mentioned above, they must also preferably fit seamlessly into the existing system and offer a long-term reliability and benefit. These requirements also define the role of aviation research: New ideas must be investigated and models developed such that the interactions with all adjacent subject areas can be taken into account. Due to the long life-cycles, this research approach must not only be applied in light of current technological requirements and regulatory frameworks, it must also consider potential future scenarios during the next forty to sixty years.

To address this complex framework, the Cluster $\mathrm{SE}^{2} \mathrm{~A}$ has structured its research units within three major Integrated Cluster Areas (ICAs):

\section{ICA A: Assessment of the air transport system}

ICA A focuses on system analysis and evaluation of the air transportation system in different future scenarios. These scenarios consider environmental, economic and social criteria simultaneously. Technology assessment includes noise exposure and life-cycle analysis.

\section{ICA B: Flight physics and vehicle systems}

ICA B conducts fundamental research on enabling technologies for sustainable transport aircraft with zero-fossil energy supply. These enablers are expected to be propulsion integration, active means of load control, drastic reductions of aircraft drag, and knowledge on integrating new functions into loadbearing structures, on new materials, and on design rules for composite structures.

\section{ICA C: Energy storage and conversion}

ICA C investigates the fundamentals of providing necessary energy and energy density required for flight. There are long-term technological prospects for full electrification of small and regional aircraft, $\mathrm{H}_{2}$-based systems for short to medium range aircraft, while long-range aircraft will continue to rely on liquid fuels. Between these distinct energy storage alternatives, there are hybrid concepts that open a new design space for adapting the aircraft to specific requirements of its mission profile and for improving overall efficiency. 


\section{Research approach and overall design methodology}

\section{A. Research approach of the Cluster}

The complex overall framework described above leads to the conclusion that research in a single field will not be sufficient to cope with the challenges of shaping a sustainable future air transport system. Instead, scientific research and fundamental technology development are needed in all relevant areas. Air travel distances range between 500 and $16,000 \mathrm{~km}$. The specific energy content of potential energy carriers also varies greatly, as do the associated energy costs. It has to be expected that the solution space of future energy supply for commercial aircraft will allow for some variation as a function of aircraft mission and hence, of aircraft segments. This explains the need to investigate a range of PTX-processes and corresponding means of energy conversion. For regional and short-range flights, avoiding conversion processes by direct electrification of future aircraft is an attractive option as far as energy density allows. Moreover, technological progress in power density of electric components will also pave the way for exploiting certain physical characteristics of the aircraft and its mission through hybrid energy architectures. Technical progress in these field calls for new knowledge gained from advanced simulations and fundamental experiments, as a sustainable commercial aircraft requires far more drastic improvements yet in power and energy densities due to first principles of flight physics.

The research area ICA B shall be used as an example to highlight some of the research results and also illustrate the linkage between the individual research units. ICA B advances fundamental research on enabling technologies for viable future transport aircraft powered by zero fossil energy. Figure 2 shows an overview of the structure in ICA B and its research units, which are focused research units that facilitate close collaboration of a number of Principal Investigators (PI) and their research projects. These research units are linked to each other by essential deliverables and data exchange platforms. Whenever possible, this data exchange is based on standardized structures, e.g. CPACS (Common Parametric Aircraft Configuration Schema), which are modified or extended to cover new types of data.

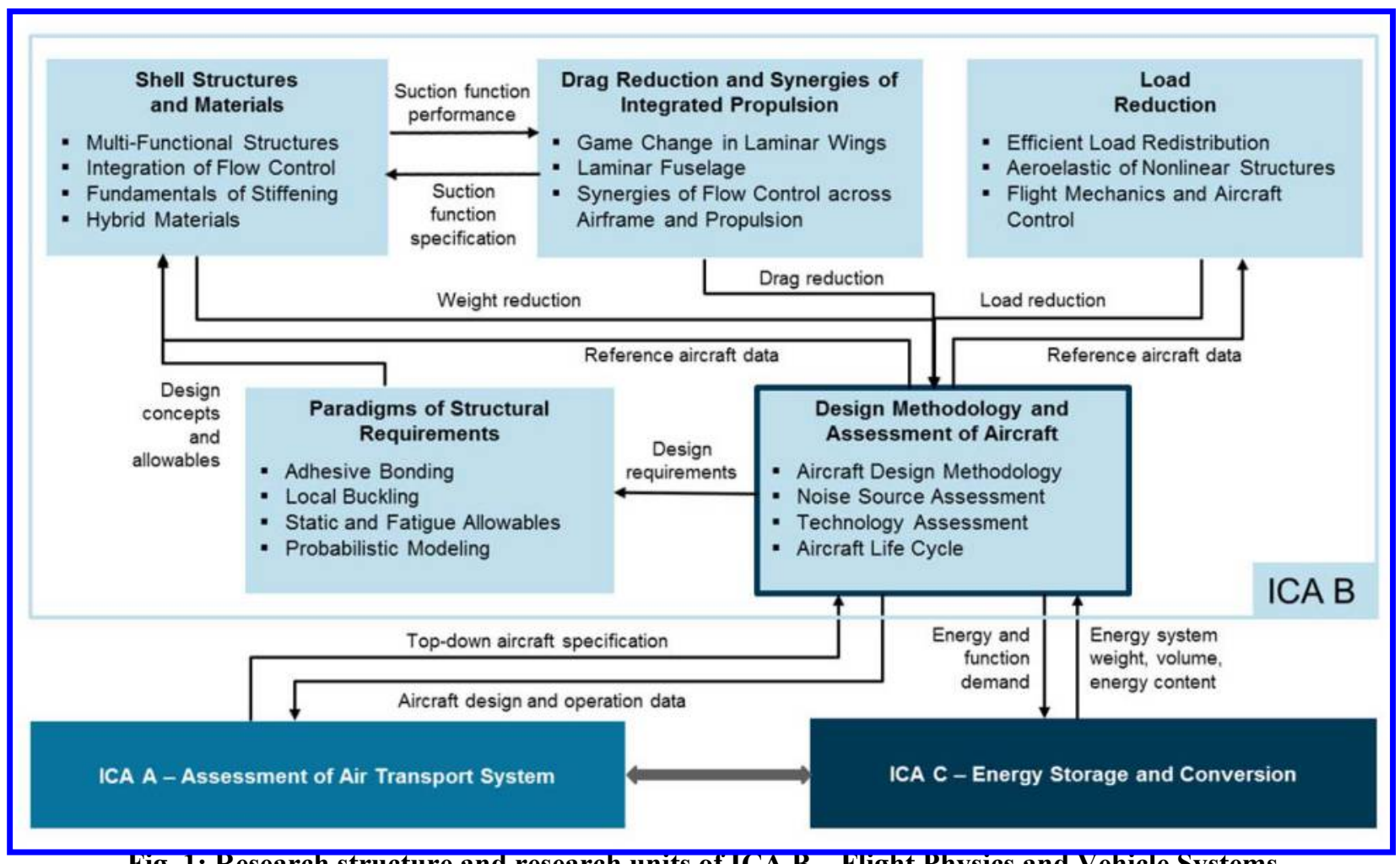

Fig. 1: Research structure and research units of TCA B - Flight Physics and Vehicle Systems 
A very important part of the research structure is the research unit "Design methodology and assessment of aircraft". This unit plays a double role within the Cluster: on one hand, it is defining top-level-requirements (TLR) as a framework or reference condition for the individual research units, while on the other hand it is collecting their individual research results - typically in the form of technological performance indicators - in order to integrate these on a preliminary aircraft design level. Such integration simulations at the aircraft level do not only facilitate the quantification of snowball effects resulting from new technologies and synergies between different technologies, they will also identify the need for configuration changes that possibly change the value of individual technologies. Similar integration platforms are also defined within ICA A (Scenario analysis and multi-criteria evaluation of the air transport system) as well as in ICA C (Design methodology for aircraft energy supply systems). A similar approach has already been used within the DFG-CRC880 "Fundamentals of High Lift for Future Civil Aircraft", see also [2], [3], and [4].

\section{B. Framework and technologies for overall integration study}

To design the reference aircraft of the $\mathrm{SE}^{2} \mathrm{~A}$ Cluster as well as to conduct overall integration studies, a multilayer aircraft design framework named ADEMAO (Aircraft Design Engine based on Multidisciplinary Analysis and Optimization) is developed, see Fig. 3.

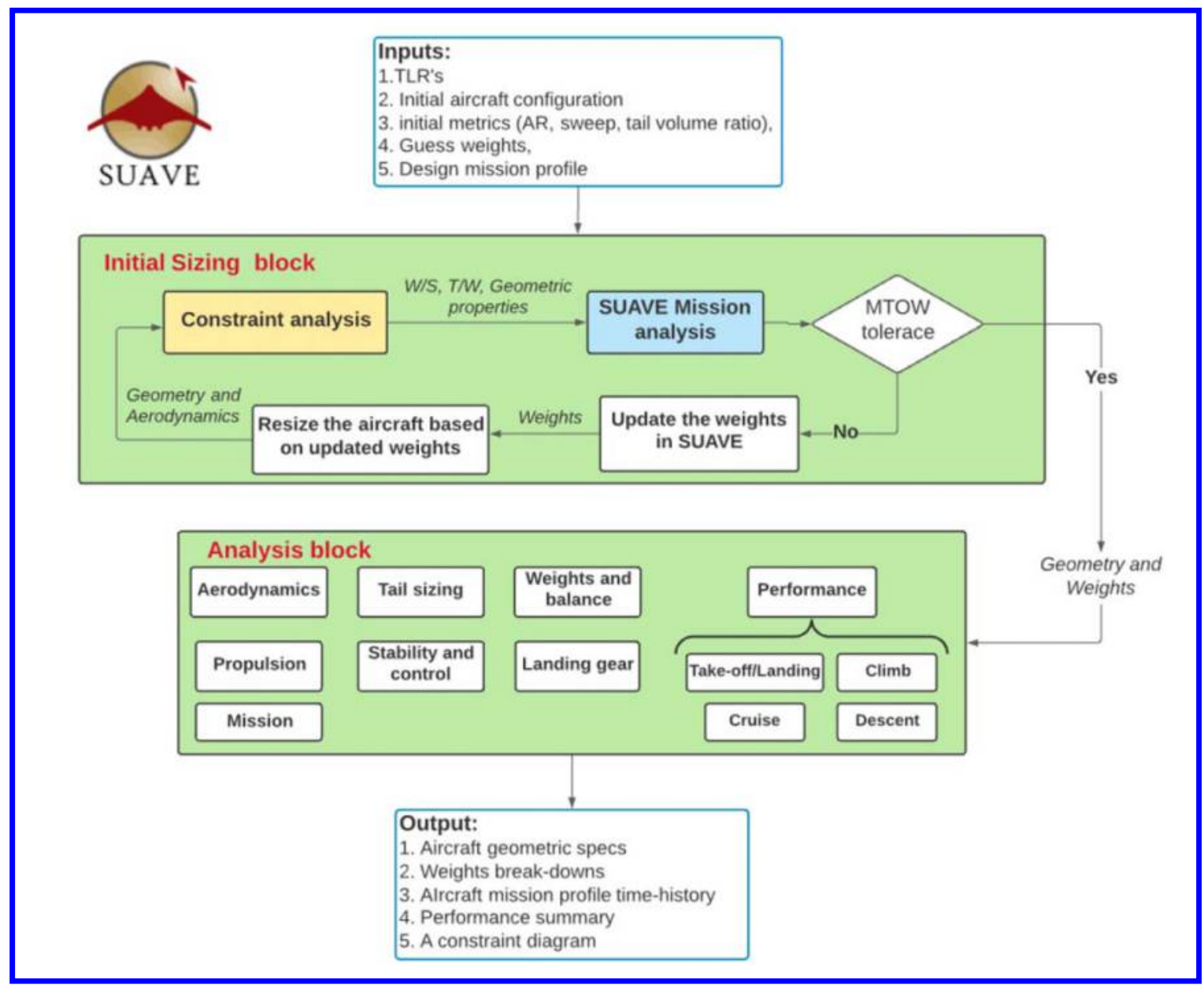

Fig. 2: Architecture of the first layer of the ADEMAO design framework.

The framework builds on the integration of four different layers:

- Layer one: An aircraft conceptual design tool, which can be used for clean-sheet design. This layer includes all the different disciplines involved in aircraft design. 
- Layer two: A surrogate modeling toolbox, which can be used to generate surrogate models based on the outcomes of different research teams and integrate it with the aircraft conceptual design tool. This layer will also enable a multi-fidelity optimization between layers one, three, and four.

- Layer three: A physics-based Multidisciplinary Design optimization (MDO) toolbox, which improves the initial design provided by the first and second layer and generates more detailed information about the design. This layer is based on medium-level fidelity but physics-based analysis, allowing us to integrate more design disciplines in a computationally affordable way than high fidelity methods. This toolbox will be used for more detailed optimization of the output of layer one, such as aeroelastic tailoring of the wing, including boundary layer suction and active load alleviation.

- Layer four: High fidelity MDO including a few main disciplines involved in the aircraft design.

The first layer of the ADEMAO framework is used for the clean-sheet design of multiple aircraft in different classes, i.e., a short-range, a mid-range, and a long-range aircraft, including the novel airframe and energy network technologies of the $\mathrm{SE}^{2} \mathrm{~A}$ Cluster. These three reference aircraft are used to quantify the influence of the combination of new technologies with new aircraft configurations on emission reduction of civil aviation. The opensource aircraft design and assessment tool SUAVE is used as the core of this layer, however, with several modifications. Figure 3illustrates the first ADEMAO layer.

For the short range aircraft design, a battery-based full electric aircraft design is considered. The TLR are assumed similar to the ATR-72 aircraft. Multiple aircraft configurations were sized and the best one, i.e., the one with minimum energy consumption, is selected for further analysis and refinement. The final design includes a clean sheet sizing, low/medium-fidelity MDO [5], and physics/based design optimization for refinement [6]. Fehler! Verweisquelle konnte nicht gefunden werden. shows the three initially designed configurations for this aircraft.
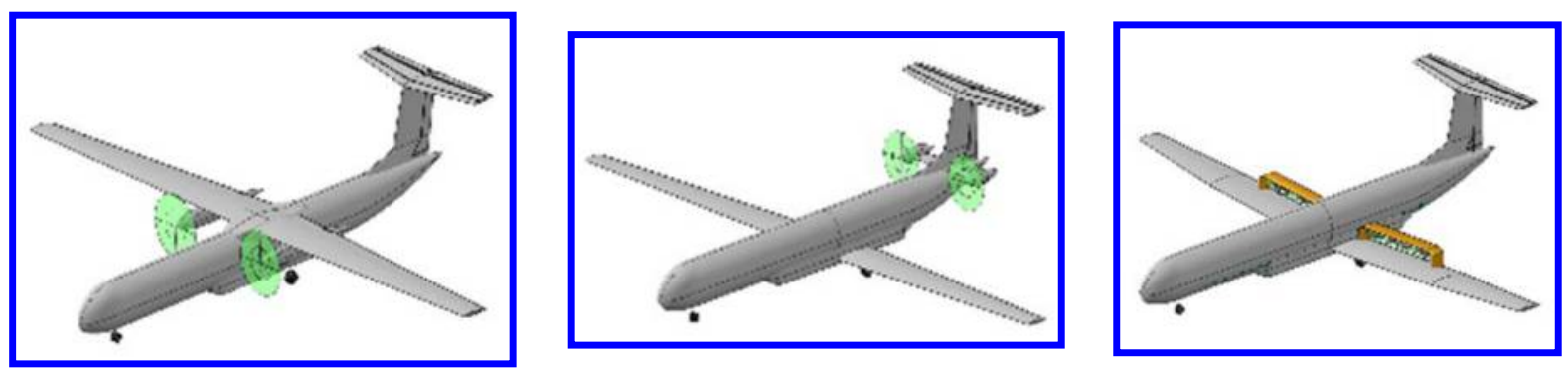

Fig. 3 Starting configurations for short-range reference aircraft design of $\mathrm{SE}^{2} \mathrm{~A}$ Cluster.

A similar approach has been used for the design of a mid-range reference aircraft. The TLRs are chosen similar to the Airbus A320. Since BLI was selected as one of the primary technologies for this aircraft, special attention was paid to propulsion integration. Two initial configurations have been conceptually designed: one with a backwardswept wing and one with a forward-swept wing, see Fig 5. The main motivation behind using a forward-swept wing is reducing the leading edge sweep angle compared to a backward-swept wing with the same mid-chord sweep angle, which is helpful for realizing natural laminar flow on the wing.

While the ADEMAO framework allows for an easy integration of new blocks for alternative energy storage as well as energy conversion on board up to the propulsor itself, it is obvious that all of the energy carriers which offer a significant improvement in terms of gaseous or aerosol emissions come with drawbacks in energy density or costs. This leads to the strong requirementof reducing the energy demand during operation as much as possible by increasing the aerodynamic efficiency and reducing the operating empty weight significantly. Based on preliminary design calculations, the suitable target for aircraft drag reduction has been identified as $50 \%$ and for structural mass reduction as $40 \%$ in order to utilize very advanced concepts for commercial air transport by 2050 . 

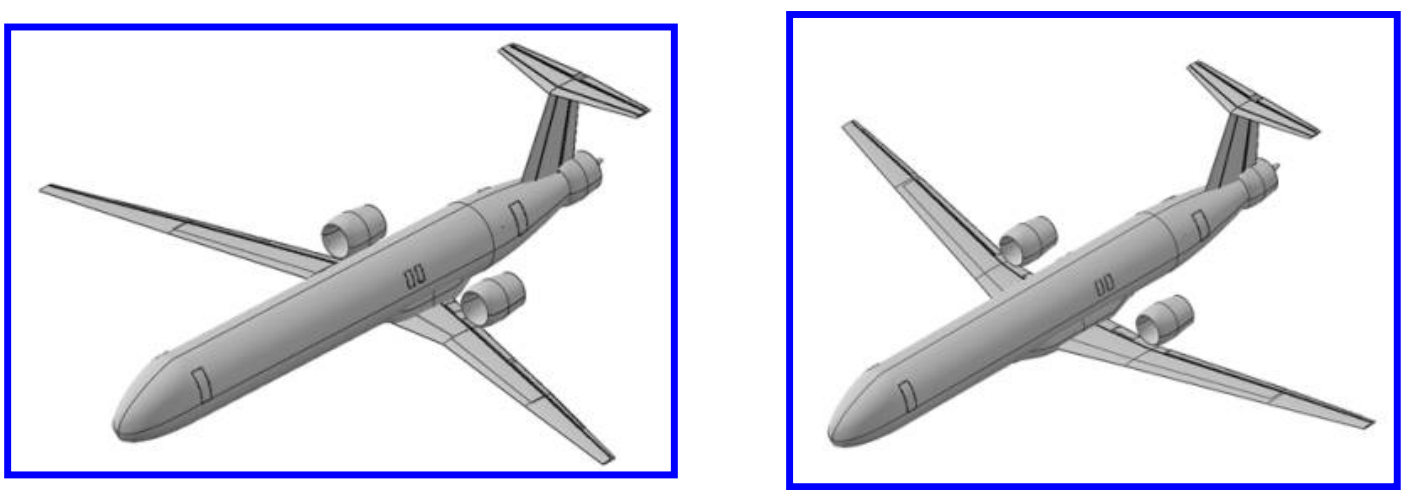

Fig. 5 Starting configurations for mid-range reference aircraft design of $\mathrm{SE}^{2} \mathrm{~A}$ Cluster.

The $\mathrm{SE}^{2} \mathrm{~A}$ Cluster has identified a set of key technologies needed to reach large improvements in drag, weight, and propulsive efficiency. While some of them do not require new knowledge, such as windowless fuselages for example, there exist high-potential technology areas where the present knowledge base is small and long-term fundamental research is needed. The general research hypothesis formulated above leads us to claim detailed working hypotheses, which call for long-term fundamental research into the following directions:

- Drag reduction by systematic laminarization of all aircraft components offers the largest potential for reducing energy consumption. It also offers large indirect gains by exploiting snowball effects on overall aircraft design level.

- Research on composite materials and function integration into Composite multi-shell structures as key structural enablers for considerable weight savings.

- New, multidisciplinary means of aircraft control to pave the way towards considerable reductions of structural weight by means of load control.

While all of these new technologies require comprehensive fundamental research to increase their TRL on an individual level, also mutual interactions and influences between technologies and towards all aspects of aircraft design have to be identified and understood. In the expectation of the $\mathrm{SE}^{2} \mathrm{~A}$ Cluster, a holistic design of an aircraft, which is optimized not only for new energy storage and propulsion solutions, but makes best use of all new technologies to reduce the energy demand during operation, will fully reflect the "form follows function" design approach and thus lead to more different configurations in the future (cf. section IV.B).

\section{Approaches for reducing the mission energy}

\section{A. Drag reduction by laminar flow control}

Aircraft drag does not only affect the flight performance of commercial aircraft; it will assume a much stronger contribution to direct operation costs in the future, due to the structural changes in energy supply economics. Therefore, the research strategy of the $\mathrm{SE}^{2} \mathrm{~A}$ Cluster views drag reduction of commercial aircraft as a major enabler for sustainable aviation. Technologies for drag reduction have always been on the research agenda. The aircraft drag comprises induced drag, wave drag, viscous drag, and miscellaneous, smaller contributions. The potentials of technologies for reducing the lift-dependent induced drag are rather limited as long as viscous drag dominates the overall drag balance. A similar statement holds for the wave drag of cruise flight at high Mach numbers. It is also known that passive and active means for reducing the friction drag of turbulent boundary layers are rather limited in their effect.

Systematic laminarization of the major aircraft components offers the largest potential for reducing aircraft drag and hence reducing energy consumption. It also offers large indirect gains by exploiting synergy effects on overall aircraft design level. This was demonstrated by recent preliminary design computations of Beck et al. [11], who assessed boundary layer suction rates and the component drag of wing, tail and fuselage for a mid-range commercial aircraft. The results indicate drastic drag reduction potentials if comprehensive laminarization of all major aircraft components is introduced. Significant is also the so-called snowball effect on overall aircraft level with respect to redesigning the wing planform and re-sizing the overall aircraft. 
Comprehensive laminarization goes far beyond designing the aircraft geometry for maximum natural laminar flow (NLF). NLF can only reduce the viscous drag of the wing and tail by about $40-50 \%$. Further drag reductions are accessible only with active flow control by boundary layer suction (BLS). We note that BLS should be applied only where absolutely needed, as the required installation of suction devices increases aircraft complexity, and adds to the required suction power on board. This constitutes the so-called hybrid laminar boundary layer control (HLFC).

The general approach towards HLFC is not new. A number of HLFC variants have been researched for several decades, and a significant number of successful tests in wind tunnels and in flight have been reported [11]. Well known problem areas are high manufacturing costs of wings with HLFC systems and their additional weight, as well as operational issues. Comprehensive laminarization of a wing as discussed above has not yet been demonstrated. The rather slow technological progress appears to be mainly caused by the interdisciplinary nature of the required engineering solutions. A major problem has always been the compliant structure design that provides the flow control function with acceptable weight.

\section{Aerodynamic design for high-aspect-ratio wings}

The aerodynamic design of HLFC on wings with high aspect ratio takes advantage of the infinite swept-wing approximation, i.e. the aerodynamic design effort focuses on identifying airfoil shapes that are best suited to yield low wing drag at low suction power input for best overall efficiency. Optimization including suction power is of particular importance for wings with comprehensive laminarization over most of their surfaces. The current research of the $\mathrm{SE}^{2} \mathrm{~A}$ Cluster aims at identifying optimized airfoil and wing shapes that sustain laminar flow on at least $80 \%$ of the chord length using BLS. A coupled optimization of airfoil shape and the suction distribution is performed to strike the right balance of the dampening effect of BLS and the effect of pressure gradients as generated by the airfoil geometry. The objective here is to minimize the total aerodynamic drag which encompasses the profile drag of the airfoil and the suction power necessary for the BLS. In addition, the airfoil must have robust performance at off-design conditions. The current optimization loop involves three blocks: (a) shape parametrization (b) optimizer, and (c) flow solver. Reference airfoil shapes and suction distribution are parametrized using Class Shape Functions (CST). Optimization is performed with the "Non-dominated Sorting Genetic Algorithm" (NSGA II) using the CST control points as the design variables. The advantage of employing the infinite swept-wing approximation is that computationally efficient flow solvers can be used such as XFOIL for subsonic flow fields or MSES for transonic flow. The present flow calculations are performed using an extended version of the boundary layer solver, that accounts for suction [12]. An $\mathrm{e}^{\mathrm{N}}$-method according to Van Ingen [13] predicts boundary layer transition for nonswept subsonic airfoils, while the stability suite LILO [14] is employed for predicting transition in cases with both, Tollmien Schlichting instabilities (TSI) and cross flow instabilities (CFI).

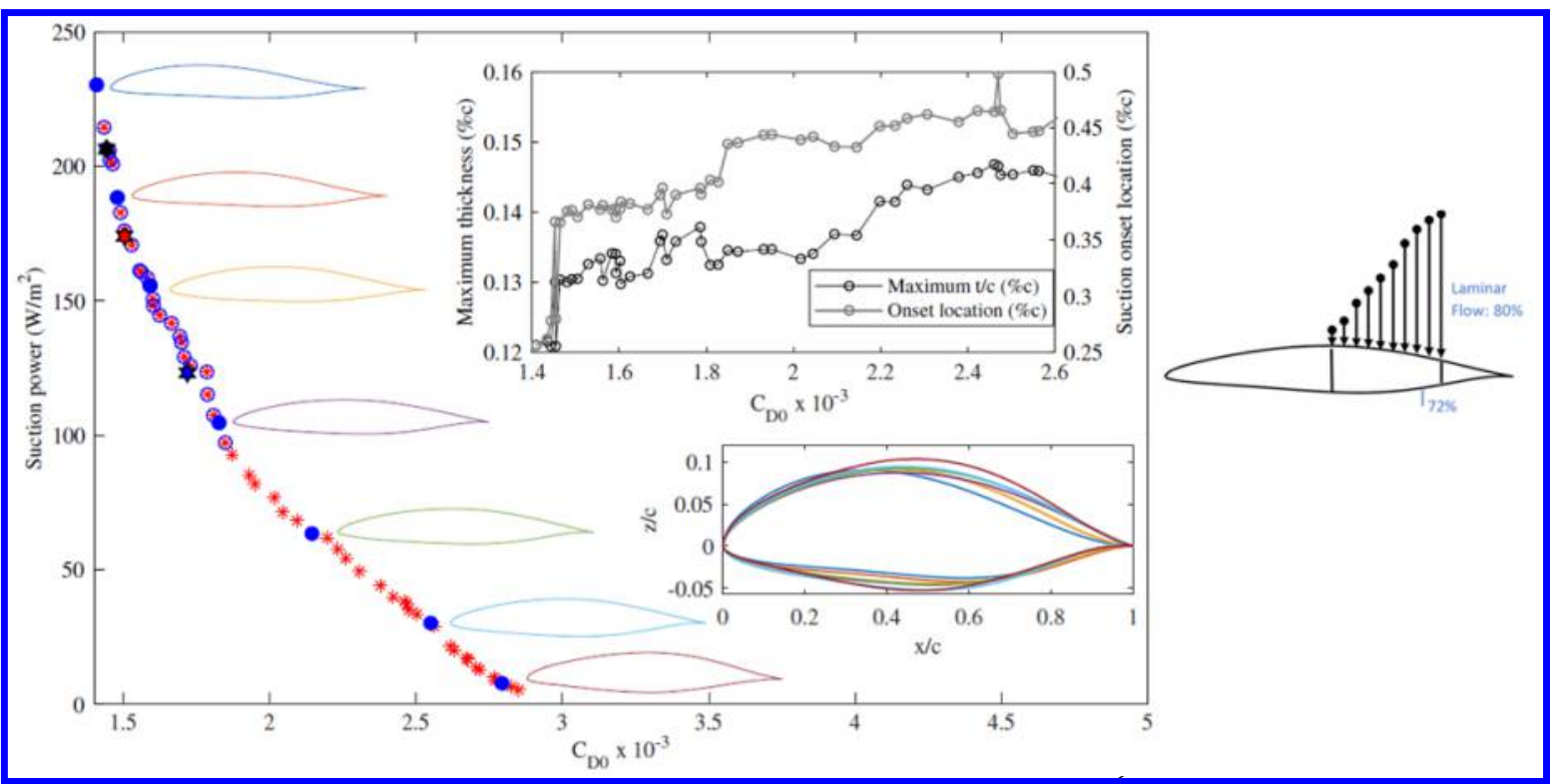

Fig. 6: Pareto front solutions for subsonic design case, $M=0.4, R e=16 \times 10^{\circ}, C_{l}=0.4$ (left) and airfoil for minimum sum of airfoil drag and suction drag (right) [15] 
Significant efforts have been spent on identifying object functions that allow the optimizer to quickly find the most favorable solutions. For subsonic flows it is effective to maximize the extent of laminar flow length for minimum airfoil drag at given suction power. This yields a family of optimum airfoils along a Pareto front that displays suction power and airfoil drag as the airfoil attributes, as shown in Fig. 6. The blue circles represent solutions with $80 \%$ laminar flow on the upper surface. The airfoil with the lowest sum of losses due to drag and suction power is also displayed on the right side of Fig. 6. It features optimized distributions of pressure and suction intensity on the upper surface, so that suction power is used at its highest efficiency. This results in $80 \%$ and $72 \%$ laminar flow on the upper and lower surfaces, respectively. As a result, HLFC reduces the airfoil drag by about $30 \%$ relative to the airfoil without suction. A thorough analysis of the influences of the allowed suction region, the intended design range of lift coefficients, airfoil thickness, and off-design behaviors is presented in Ref. [15].

The design space is much more complex at transonic flow, since the optimizer has to deal with wave drag, viscous drag, and suction drag. The wing sweep angle can be used to mitigate wave drag for a given lift coefficient and thickness, but only at expense of suction at the nose to avoid transition due to CFI. One of the cases studied until now assumes a wing design with sweep angle $22.5 \mathrm{deg}$, which is typical for mid-range aircraft. In this case the multi-objective function for the optimizer is minimum pressure drag (including wave drag and suction drag) and maximum laminar region. Transition from laminar to turbulent flow is dominated by CFI (increased with sweep angle), and TSI (increased in adverse pressure region). Therefore, the suction is applied in the LE region up to $20 \%$ chord, and the optimizer shapes the airfoil to extend the laminar region up to the shock as shown in Fig. 7. The Pareto front (not shown here) delivers the airfoil with minimum total drag. The solution has a favorable pressure distribution up to the shock. HLFC extends transition to $64 \%$ on the upper surface, and on the lower surface is laminar up to $49 \%$, while the optimizer could not find a lower surface with laminar flow without suction for the given Mach number. The computations show that the optimizer seeks to provide the required lift by shaping the forward portion and the rear loading of the airfoil along with fulfilling the two-N-factor transition criteria with respect to TSI and CFI. The drag breakdown indicates that boundary layer control reduces friction drag and form drag of the airfoil. More study cases are presented in Ref. [16]. The huge design space of airfoils for swept wings still needs further exploration, e.g. with respect to the potentials offered by combining weak shocks at the upper surface with suction in the adverse pressure gradient region.
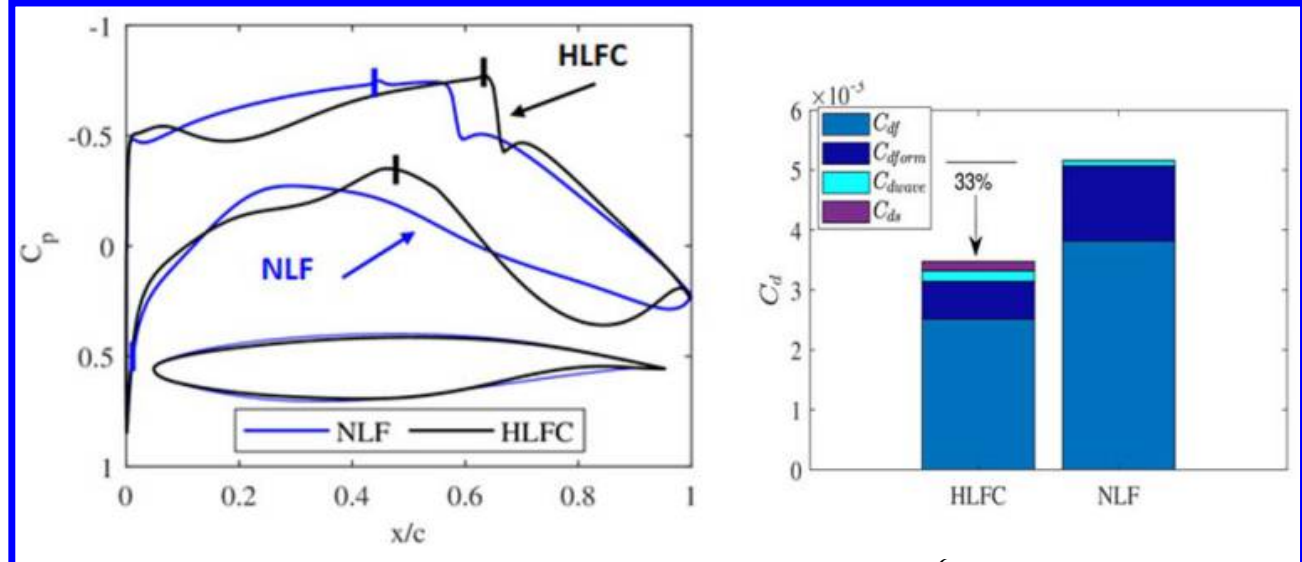

Fig. 7: Pressure distributions for transonic design, $M=0.78, R e=30 \times 10, C_{l}=0.55$ and drag break down

For HLFC systems a suited design of the suction flow is key to taking advantage of optimized suction distributions. This is facilitated by sucking the external near-wall flow through the porous skin into some collector duct that has pressure loss due to flow resistance. To drive the air through the porous skin, a pressure difference is applied between the interior and the outer surface of the skin design. We note that recent improvements in metallic wing surface materials and the technologies capable of machining them have resulted in good quality of micro-holes perforated materials. Nevertheless, the substructure below the surface has to provide a tailored pressure distribution along the streamwise direction of the wing surface so that the intended suction velocity is obtained. Figure 8 displays the solution approach for this task which is denoted as xHLFC. 


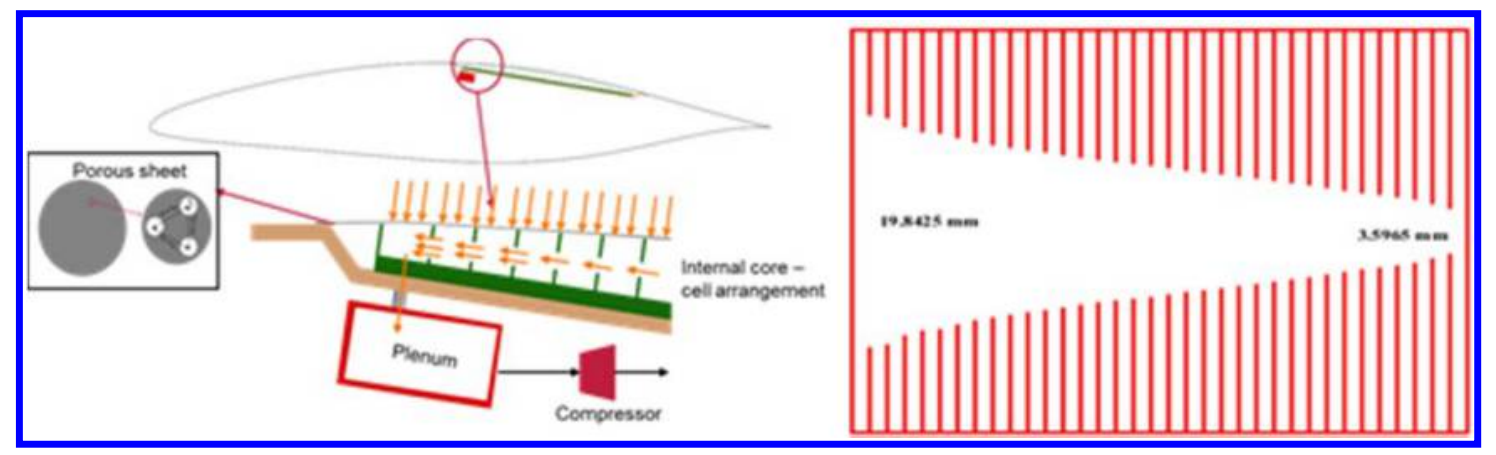

Fig. 8: Design concept of suction flow design and typical sizing data of suction buffer holes for for short range reference aircraft of $\mathrm{SE}^{2} \mathrm{~A}$ Cluster

The aerodynamic design of such function integration is the task of the software "Actual Suction Power Calculation Tool" (ASPeCT) [17]. ASPeCT starts by providing dimensional design input, as derived from the aerodynamic design of the outer flow, and from aircraft wing design data. Using a permeability model of the perforated wing surface, the internal core is discretized into cells that are connected by holes. The connected cells constitute a buffer that connects to the plenum by a throttle hole. Cell and hole sizing are iterated until a certain tolerance to the ideal pressure differential is fulfilled. Figure 8 displays a typical result of this sizing for the buffer for the wing of a subsonic aircraft. Hole diameters vary from 3.6 to $20 \mathrm{~mm}$ while the length of the 38 cells in streamwise direction is kept uniform at $25 \mathrm{~mm}$. The results of that design are then expanded for the finite wing. This leads to the sizing of the required mass flow and suction power of the overall wing.

A current research interest is to study different concepts of inner core based on a range of representations of flow resistance. With the help of 3D printing technology different concepts of the internal sandwich core structure to support the porous sheet are being explored. One research target is to allow for tailored flow resistance in Triply Periodic Minimal Surface Structures (TPMS) and to derive suitable reduced order models of the flow through the core to facilitate aerodynamic design. Figure 9 shows three different concepts of the internal design. Flow bench tests are carried out to study the pressure distribution (loss) based on the design variables.
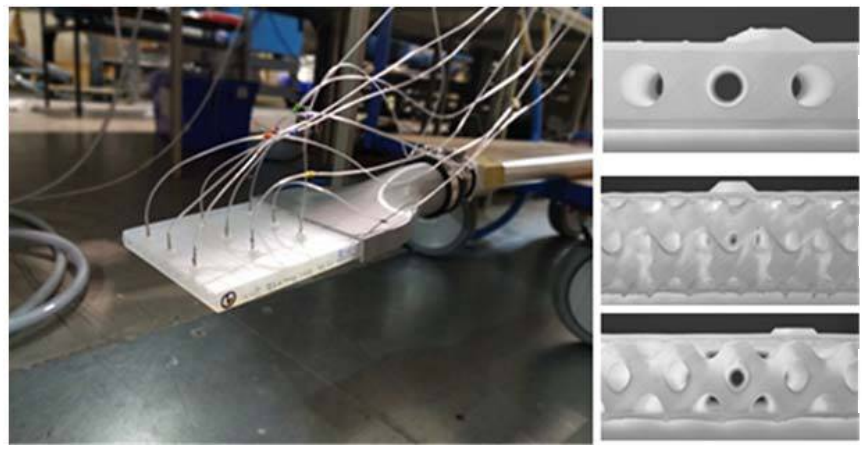

a) Base-Channel core

Fig. 9: Inner suction core concepts and test bench for flow resistance measurements

Comprehensive laminarization of aircraft wings and bodies using new technologies in aerodynamics and structures makes thorough technology validation necessary. The $\mathrm{SE}^{2} \mathrm{~A}$ Cluster follows a stepwise approach to validation that takes technical needs and available research infrastructure into account. The approach comprises fundamental transition studies on a flat plate, intermediate experiments of a research airfoil in a medium-size wind tunnel, and finally, thorough technology testing in a large world-class wind tunnel facility.

The flat-plate tests aim at measuring the quantitative effect that suction has on laminar turbulent transition and validate the ability to predict that effect by computation. They also address the possible problem of aerodynamic roughness created by suction through micro holes of different size. The flat-plate tests further aim at quantifying porous-skin flow resistance under realistic outer flow conditions. xHLFC experiments on a dedicated research airfoil will verify the integrated design capability of porous skin and sandwich core. They also address the problem of rapid boundary layer transition in flow regions with strong adverse pressure gradient, downstream of the chosen suction interval. This is a new research question associated with comprehensive laminarization of the wing towards the 
trailing edge. Finally, the airfoil tests will demonstrate the low drag of an airfoil with $80 \%$ laminar flow. The third validation step aims at fulfilling the needs to test at elevated Reynolds numbers and to simulate the aerodynamic effects of wing sweep. A suited facility for that purpose is the DNW-NWB 3 m wind tunnel.

The $\mathrm{SE}^{2} \mathrm{~A}$ Cluster has recently completed first flat-plate experiments. Here, the modal growth of TollmienSchlichting waves causes large, primary disturbances of the boundary layer. This sets the stage for break-down to turbulence. With suction, boundary layer transition can be delayed by attenuating or suppressing the growth of these primary disturbances. The experiments of the flat plate with a suction insert were conducted in the low-speed wind tunnel MUB of the Technische Universität Braunschweig. Reynolds number varied between $4-8$ Million. The experiments yielded valuable experience on the design of compliant suction chambers, and indicate fluid mechanical constraints on the allowed suction holes as well as suction effects on critical $\mathrm{N}$-factors [18].

\section{Compliant Structures}

This section aims to develop a structural concept for active suction structures. Following a structural design methodology, the authors separate the suction panel into its basic functional elements. Structural design solutions for all functional elements together form the suction panel's design space. The design space allows to derive a discrete suction panel designs by selecting specific solutions for each functional element. Existing structural design solutions for suction panels can be shown and compared using the suction panels design space. However, including new elements and technologies, such as perforated carbon fiber-reinforced plastics (CFRP) and additive manufacturing (AM), allows structural design solutions beyond state-of-the-art technology.

The suction panel consists of a perforated suction skin (A) supported by a core structure (C) and a backbone structure (E). The core structure is simultaneously used as a duct for the drawn in air and to support the suction skin. Both interfaces (B, D) combine the single components into a lightweight sandwich structure, the suction panel. The main functions of the panel are: (1) continuous suction in order to stabilize the disturbed boundary layer, (2) maintaining the airfoil's shape under structural and aerodynamic loads and (3) supporting the wing skin against stability failure. Figure 10 shows the cross section of a xHLFC suction panel as defined by Traub et al. [29].

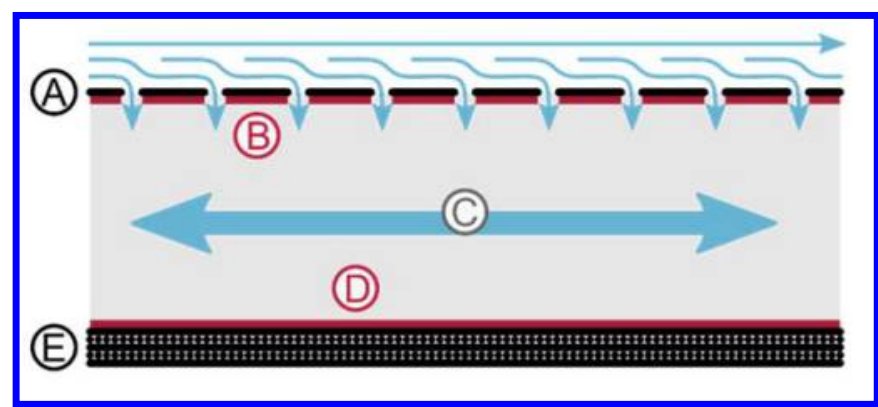

Fig. 10: Cross section of an $x H L F C$ suction panel:

(A) perforated skin, (B) skin-core interface, (C) core structure, (D) panel interface and (E) backbone structure

Within the suction panel design, the task is to minimize the area of contact between skin and core in order to reduce hole blockage, to realize a core structure that is efficient from an aerodynamic and structural point of view, and to make the best possible use of the material by means of effective stiffness distribution. AM technologies offer huge potential regarding function integration and integral part design. Therefore, the focus of this section is to exploit the possibilities of AM in the suction panel design. A generic design approach allows to compare and assess different design solutions for suction panels. Figure 11 shows the design space for XHLFC suction panels including solutions only feasible using AM. The design space is created by the individual solutions for the suction panel components. The five components of the structure are porous skin, skin-core interface, core structure, panel interface and wing skin. The partial solutions include design, material and manufacturing process. A suction panel design can be obtained by combining partial solutions for each suction panel component. Therefore, this approach contains more than 2500 design solutions including existing solutions for HLFC and LFC suction panels. 


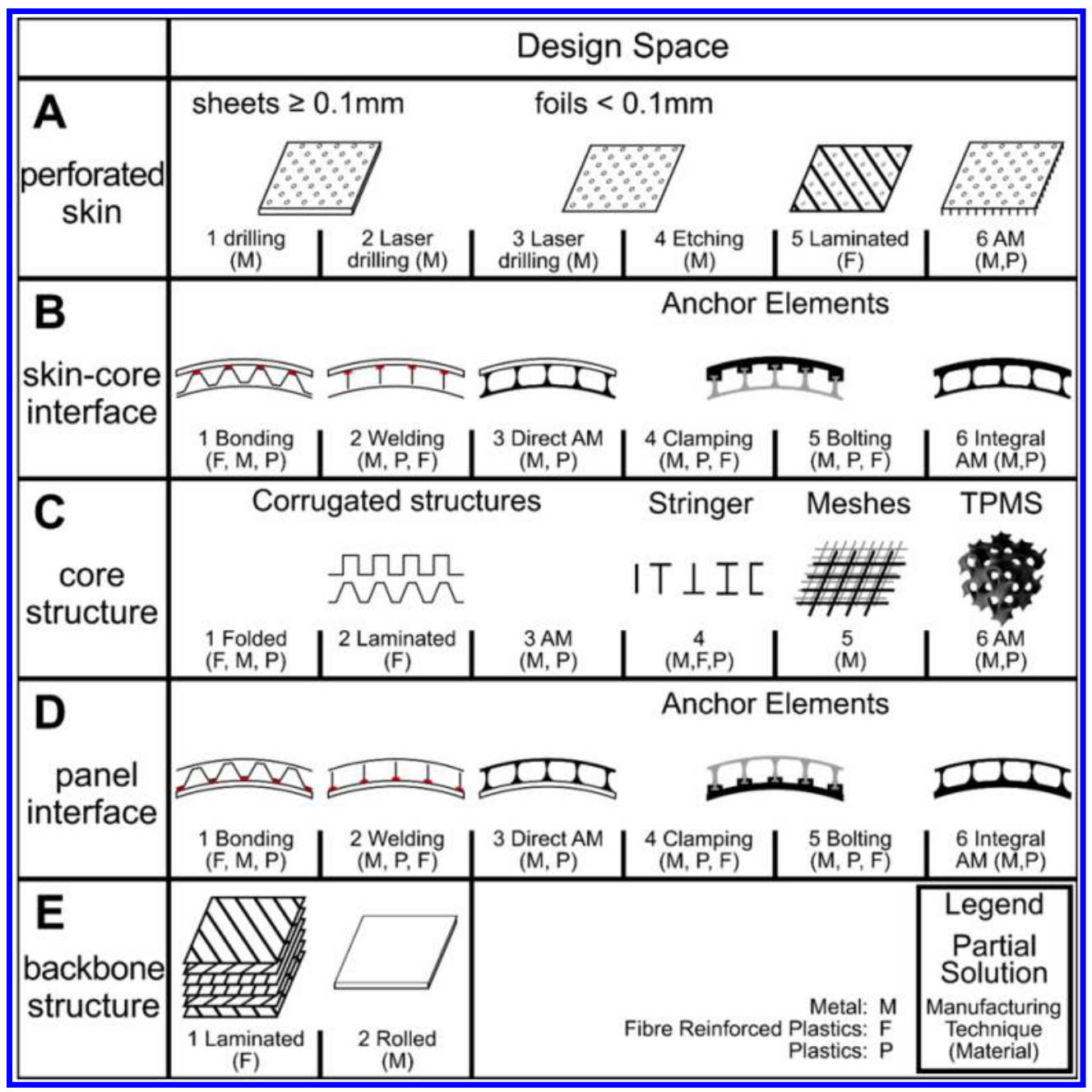

Fig. 11: Generic design space for an active suction panel including partial solutions for AM

The core is a so-called triply periodic minimal surface structure (TPMS), which consists of small, recurrent volume elements with 3D surfaces with zero mean curvature at every point. Therefore, the TMPS's surfaces are free of lateral forces and act mechanically as membranes. Typical TPMS structures like Gyroid or Schwarz Primitive (cf. Fig. 9) are promising approaches for core structures, since they provide both a lightweight but dense structure [35] as well as a channel system enabling internal air flow. Complex, integral structures like TPMS can only be realized with AM in terms of production technology and cost-effectiveness.

The outer suction surface as well as the TPMS core are manufactured integrally by AM-technology, without any assembly. This element is bonded to the load-carrying wing skin, which is made from CFRP. This way, a lightweight structure resilient to stability failure is generated. The minimal surface concept suction panel can be printed on various 3D printing systems. Selective laser melting (SLM) printers allow to use engineering materials such as stainless steel or titanium creating structures resistant to most environmental impacts, e.g. UV radiation or corrosive media. However, stereolithography (SLA) and selective laser sintering (SLS) are a lightweight alternative to SLM, producing high quality thermoset and thermoplastic parts. Figure 12 shows an SLA printed xHLFC suction panel demonstrator with printed perforated skin and a gyroid core structure.

Currently, this concept is under investigation for its mechanical and aerodynamic performance. Aim of the investigation is the achievable surface quality, shape retention and reproducibility of the suction holes. The perforated suction skin is evaluated by flow meter tests and the TMPS are evaluated by flow bench tests in comparison to the corrugated cores. The structural performance of the TPMS is also investigated and the limits of the AM technology in terms of manufacturing parameters such as minimum wall thicknesses are determined in order to realize efficient lightweight structures. 


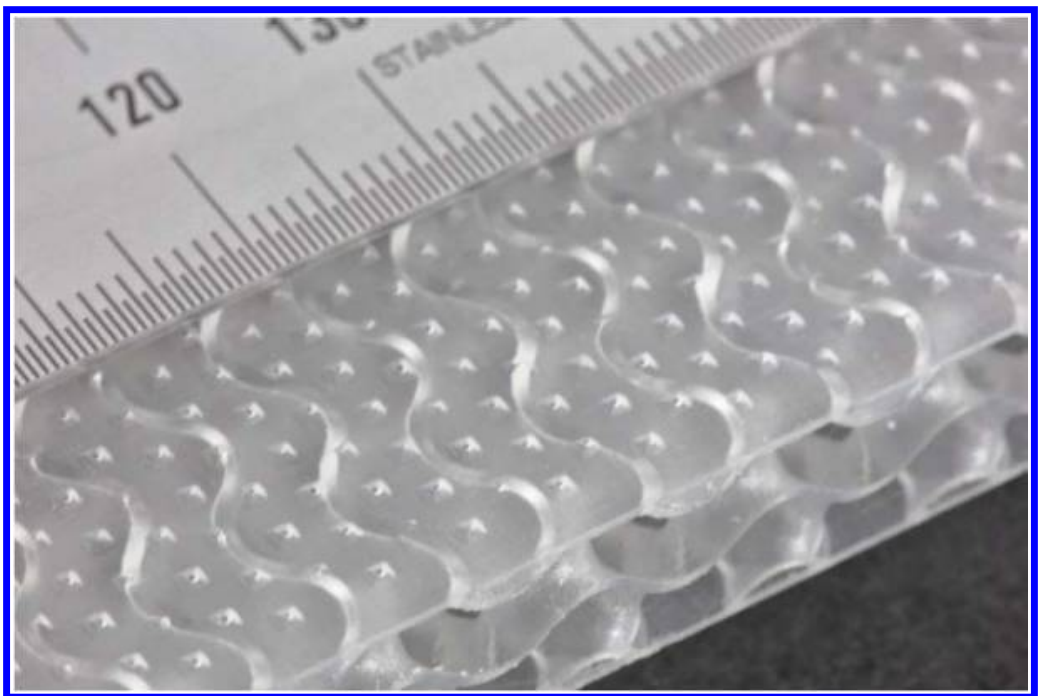

Fig. 12: Additive manufactured suction panel demonstrator (Gyroid, skin porosity 1\%)

\section{Boundary Layer Ingestion}

Closely coupled to drag reduction by actively controlling the boundary layer development on ideal surfaces of the aircraft is the technology of boundary layer ingestion which combines drag bookkeeping methods of the aircraft with propulsion integration and propulsive efficiency assessment. Although BLI and wake-filling is easy to be described from a phenomenological perspective, a sound prediction of savings is a rather complex issue. This is due to the complex nature of 3D boundary layer flow in combination with a wide range of ingestion concepts, e.g. fully embedded engines vs. partially embedded concepts of different circumferential as well as radial extension. In addition, for a feasible prediction of the PSC (Power Saving Coefficient), which describes the reduction in required propulsive power to balance the total drag, also the impairment of the fan by the inhomogeneous inflow must be taken into account. Gunn and Hall describe a consumption reduction of up to $15 \%$ with a reduction of the stage efficiency of the fan of 1-2\% [8]. In own studies within $\mathrm{SE}^{2} \mathrm{~A}$ based on a parallel compressor model [9] applied to the blended wing body and using the performance map of a fan designed within CRC880 [10], a PSC of 5-6\% was identified. The main difference was introduced by higher efficiency degradation for the fan in the local boundary layer ingesting areas. Here, the fan efficiency in the disturbed sector was about $3 \%$ lower than that of the undisturbed sectors, resulting in an average efficiency reduction of about $1 \%$.

\section{B. Weight reduction by load alleviation}

The $\mathrm{SE}^{2} \mathrm{~A}$ Cluster investigates a range of approaches towards significant load alleviation. The objective is to find solutions to significantly reduce limit loads, as this generates large reductions of Operating Empty Weight. The general idea of load alleviation has been well known for decades. Active and passive approaches may be followed and most likely need to be combined in the end. Significant design space is offered by the concept to change the wing load distribution in a passive way by taking advantage of tailored stiffness of the outer shell, while the nonlinear response of the inner-wing structure opens an alternative path to desired shape response. The first section of this sub-chapter reviews recent efforts on passive approaches to achieve this goal, followed by a second section that presents active measures of load alleviation.

The $\mathrm{SE}^{2} \mathrm{~A}$ Cluster has defined its mid-range reference aircraft as its initial, joint application scenario for load alleviation. The current research focuses on wing load reduction and its primary objective to reduce wing structural weight. Note that all approaches of load alleviation generate certification issues; these should be addressed in detail once technically sound concepts are identified.

\section{Passive load alleviation}

Nonlinear stiffness of wing structures can be employed to gain load reduction. The way non-linearity is achieved distinguishes different concepts followed in the $\mathrm{SE}^{2} \mathrm{~A}$ Cluster from each other. One approach investigates the use of tailored material non-linearity. The idea is to take advantage of a nonlinear softening approach that reduces stiffness above $1 \mathrm{~g}$ load cases. That allows to provide minimum induced and wave drag during low-load cases, while lift distribution is significantly shifted inboard at high loads [19]. In addition, viscoelastic damping is 
investigated as a further means of load reduction. Here the concept of Constrained Layer Damping is advanced, where the viscoelastic material is constrained between the host shell structure and a stiff face layer. Optimization of the geometrical parameters has yielded significant modal reductions at moderate gains of overall structural mass [19]. These initial results justify future, more detailed analysis. Identification of promising aeroelastic tailoring technologies is a further objective of the Cluster. This involves a multidisciplinary design process that includes aeroelastic analysis of the shell including its stiffeners. Hereby, detailed structural constraints like strength and stiffness are considered and sensitivities of structural design parameters regarding mass, bending and twist stiffness are identified based on structural optimization. Passive load alleviation through stiffened panels in post-buckling regime aims to identify meaningful applications on wing covers. In order to achieve that, a new methodological approach for analysis and sizing is developed and applied that allows analysis of unconventional structural designs.

An alternative approach exploits the concept of morphing wing sections, such that the reduced aerodynamic load at the outer wing area leads to a reduced wing root bending moment. This research also employs a nonlinear passive load alleviation, which means that the wing remains stiff up until a defined critical load level above $1 \mathrm{~g}$ flight and deforms significantly at loads above that level. To achieve this, selected structure components with a strong non-linearity such as observed with buckling are exploited. The non-linearity begins at the critical load level and changes the overall stiffness distribution. This concept is investigated for a transonic transport aircraft wing. Structural and high fidelity aeroelastic analyses in the time domain are employed.

A two-dimensional concept for load alleviation in wing sections produced by nonlinear structural response is to include a stiffener into the inner structure of the airfoil, which buckles at the critical load due to compressive stress. The resulting deformation of the airfoil leads to reduced camber and lift. This concept is investigated using nonlinear numerical models of fluid and structure with the coupling environment ifls [20]. More details of the investigation are found in Ref. [21]. The nonlinear mechanism is depicted by the deformation plots of quasistationary analysis in Fig. 9 (left). Here, the DLR-F15 supercritical airfoil is simulated at $M=0.78$, corresponding to cruise condition of the $\mathrm{SE}^{2} \mathrm{~A}$ medium range reference configuration. With a freestream angle of attack required for cruise lift, the flexible aluminum structure deforms very little and the lift coefficient decreases by $8 \%$ only, from 0.376 to 0.343 , compared to a fully rigid structure. With the angle increased by $1.8 \mathrm{deg}$ representing increased load, the structure strongly deforms, thereby reducing the lift coefficient by $75 \%$. This is even below cruise $C_{l}$, which indicates the strong load reduction in stationary scenarios like maneuver loads. This can be exploited for redistribution of lift along the span to alleviate maneuver loads, e.g. for pull-up maneuvers. Gusts, on the other hand, are dynamic events, so the unsteady aerodynamic and structural response in the time domain have to be taken into account. Figure 13 (right) shows the response of the lift coefficient in a vertical gust with a wave length of $214 \mathrm{~m}$ and a vertical velocity of $10 \mathrm{~m} / \mathrm{s}$. The lift coefficient increases until the structure deforms, which is obviously attenuated by inertia forces. The load increment is reduced by $61 \%$ compared to the rigid airfoil. In shorter gusts the alleviation becomes less effective because of the short response time available. At this point it appears that this approach towards passive gust load alleviation represents a complex design problem of 3D light-weight wing structures

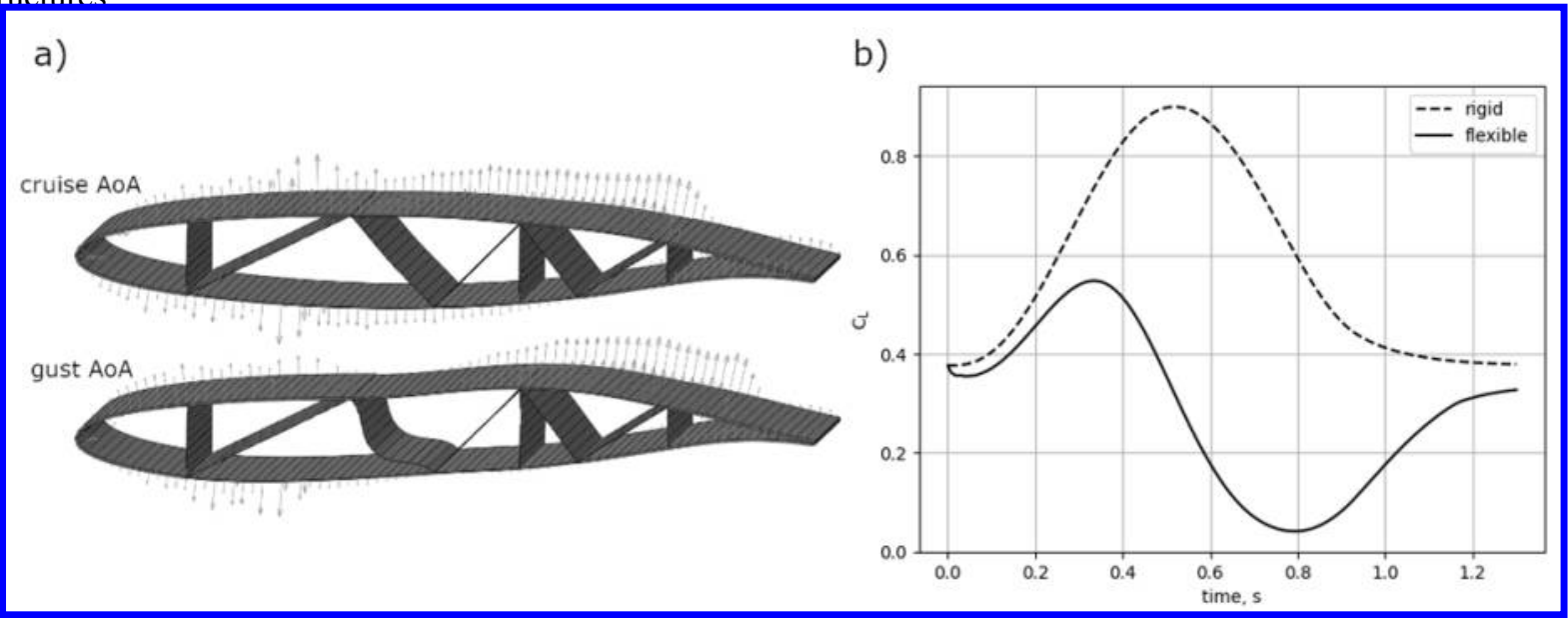

Fig. 13: Load alleviation by buckling of the inner airfoil structure. a) Quasi-stationary response to different flow angles. b) Encounter of a $214 \mathrm{~m}$ gust with the flexible airfoil concept compared to a rigid airfoil. 


\section{Active load alleviation}

Apart from the passive means to reduce dynamic loads and their potential effects on the required wing structure, there are active methods that have the potential for high levels of load alleviation and therefore substantial weight and fuel savings. State-of-the-art implementations on aircraft are typically based on sensors that detect gusts through changes in wing accelerations and structural strains and employ the available control surfaces such as ailerons to temporarily reduce wing lift locally, therefore partially counteracting the gust-induced dynamic loads $[21$, . Further improvements of the active load alleviation system potentially offer extensive reduction of dynamic wing loads, therefore enabling further weight savings and emission reductions.

The current approach for active load alleviation in $\mathrm{SE}^{2} \mathrm{~A}$ integrates the components and technologies developed in different Cluster projects into a single aeroelastic and flight mechanics aircraft model, as illustrated in Fig. 14 and described in more detail in [24]. The model is of fairly low order and comprises several hundred to a few thousand states, making it suitable for real time applications. The aircraft model includes structural dynamics, unsteady aerodynamics, and reduced-order models of advanced actuation (e.g. control surfaces or novel actuators) and sensor systems, as well as a simulation of the flight control computers with various functions, such as basic flight control laws, autopilot modes, and active load alleviation based on feed forward control.

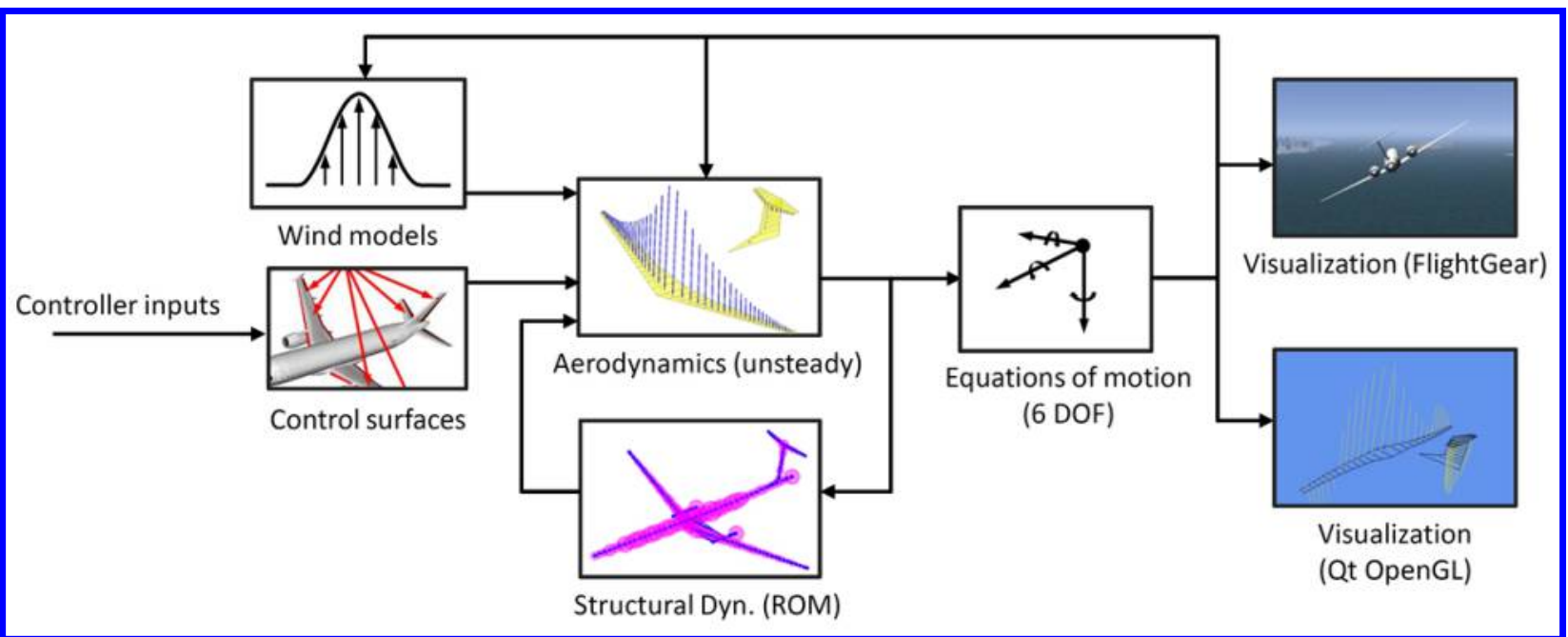

Fig. 14: Aeroelastic and flight mechanics model for active gust load alleviation, [24]

The aerodynamics model is based on the vortex lattice method coupled with nonlinear airfoil data to consider compressibility and actuator effects. The airfoil data is calculated through CFD simulations and a reduced-order model is derived for fast interpolation of load coefficients. Unsteady aerodynamics effects are described for spanwise-independent wing and wake segments, resembling a strip theory approach. A continuous state space model from Beddoes and Leishman [25] covers compressible unsteady airfoil aerodynamics, while the Beddoes and Leishman dynamic stall model [26] resolves viscous effects occurring during gust interactions, which are considered based on typical wind models like the 1-cos gust, and Dryden and von Kármán wind turbulence models. The aerodynamics model is directly coupled to the structural dynamics of the flexible aircraft, which are considered by a reduced-order model that includes the structural eigenmodes with the lowest frequencies.

On the actuator side of the active load alleviation system, both a classical approach based on primary control surfaces like trailing edge flaps, as well as a novel approach based on fluidic flow actuation devices are studied [27]. The fluidic devices have the potential for faster and therefore more complete load reduction, while allowing optimal implementation along the wing span independent of the primary control surfaces. A promising candidate for such a fluidic flow actuation system is a dual-slot tangential air jet over an elliptical trailing edge. This actuator leverages the Coanda effect to entrain and deflect the outer flow through relatively small jet mass flows, thereby effectively controlling airfoil lift. A recent wind tunnel test investigated the performance of this actuator in terms of its load control authority (lift, drag, and pitching moment) and actuation speed [28]. A sketch and detail photograph of the wind tunnel model are shown in Fig. 15, depicting the internal pressurized air system feeding the two slots on the upper and lower side of the model. The model has a chord length of $c=0.5 \mathrm{~m}$, span of $1.3 \mathrm{~m}$ and two continuous slots of $0.41 \mathrm{~mm} \pm 0.028 \mathrm{~mm}$ height along the full span, issuing highly uniform air jets over the rounded Coanda surface. 


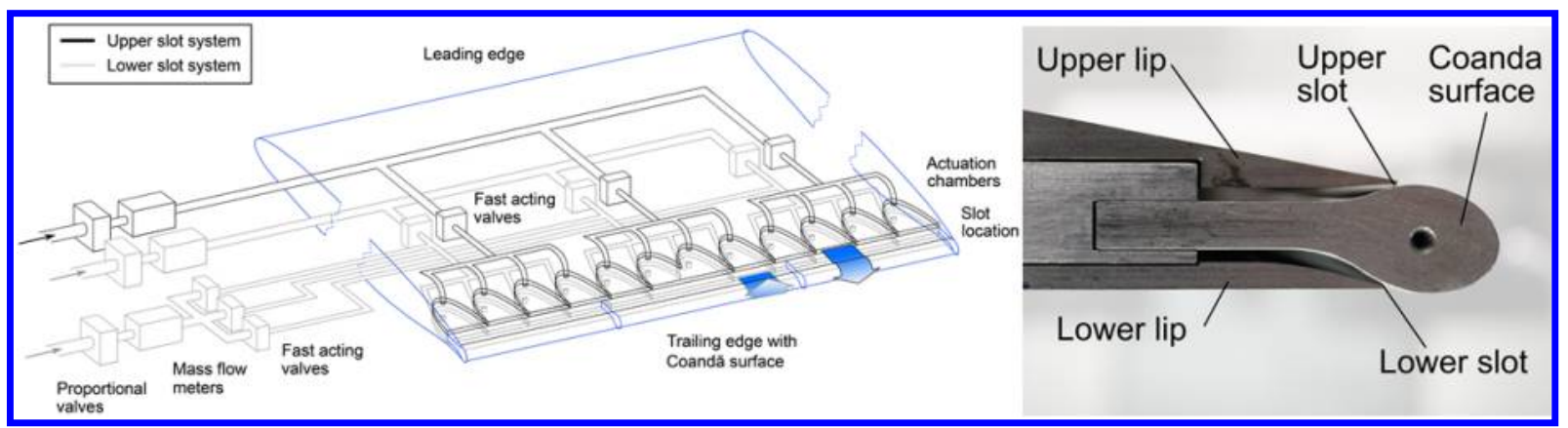

Fig. 15: Wind tunnel model with dual-slot Coanda actuator for active load alleviation, modified from [28]

The control authority of the Coanda actuator over airfoil lift is illustrated in Fig. 16. The left graph depicts relative total pressure in the actuation chambers upstream of the slots over non-dimensional time $t^{*}=\mathrm{t} \cdot \mathrm{U} \infty / \mathrm{c}$ after valve activation, with a freestream velocity of $U_{\infty}=50 \mathrm{~m} / \mathrm{s}$. The corresponding change in lift coefficient $\mathrm{cl}$ is plotted in the graph to the right, based on wind tunnel balance and integrated surface pressure measurements. The presented data highlights both the high control authority of the system with a $\Delta \mathrm{cl}$ of 0.46 between baseline performance and load control setting, as well as a fast actuation time of $t^{*}=1.165$ or $11.6 \mathrm{~ms}$ for the full load change, which is significantly faster than the onset time for the shortest gust typically encountered in flight $\left(t^{*}>2\right)$. The presented data highlights the potential of active load control systems based on fluidic jet actuation. Consequent derivation of reduced-order models is under way for implementation of these results into the previously described flight mechanics model and evaluation on a system level
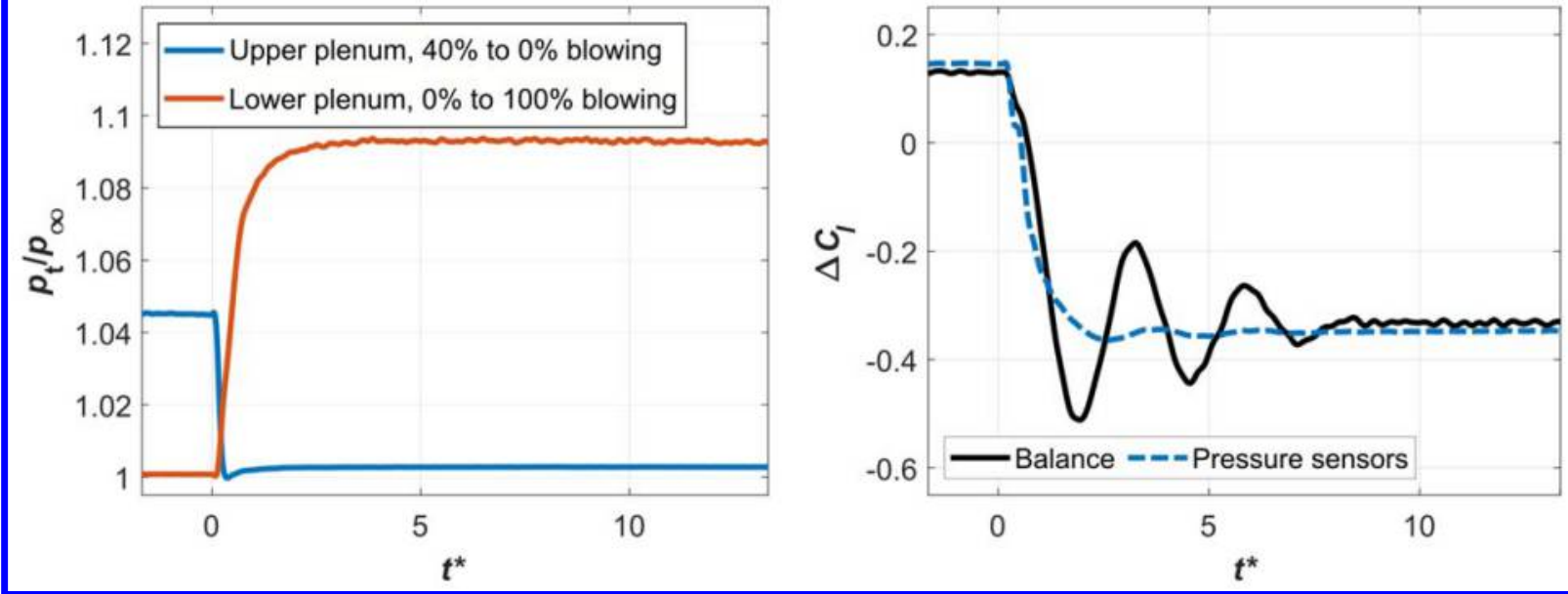

Fig. 16: Impulsive jet activation from a case close to baseline performance to full load alleviation [28]

The selected solution approach and especially the feed-forward control design of the gust load alleviation heavily rely on a number of nonlinear and coupled model-based descriptions of the flight dynamics, sensors, and actuators. It is therefore crucial to quantify their uncertainties to achieve a robust controller design, systematically assess system reliability, and determine the influence of external disturbances, sensor errors, and variations in model parameters on the actuator response or the entire load alleviation system. An example for the uncertainty propagation from the lidar-based gust detection model to the corresponding wing loads is shown in Fig. 17. The graph to the left depicts a Bayesian estimation (orange line) of the velocity distribution of a vertical gust (blue line) upstream of the airplane, including a gray credible interval that indicates the uncertainty in the prediction. Using the estimated wind profile as input for a robust control framework, the propagation of model uncertainties can be studied through the aerodynamics and structural dynamics models to the resulting wing loads, shown in the graph to the right of Fig. 17. In this graph, the non-alleviated loads of the aircraft subject to a discrete gust are compared to the loads resulting from a lidar-based feed-forward gust load alleviation controller subject to uncertainties in the estimated wind field. As illustrated in this example, the uncertainty quantification plays an important role in determining the sensitivity of the aircraft model with active load alleviation against uncertainties of model parameters and sensor inputs, therefore leading to improved reliability and robustness of the developed active load alleviation concepts. 


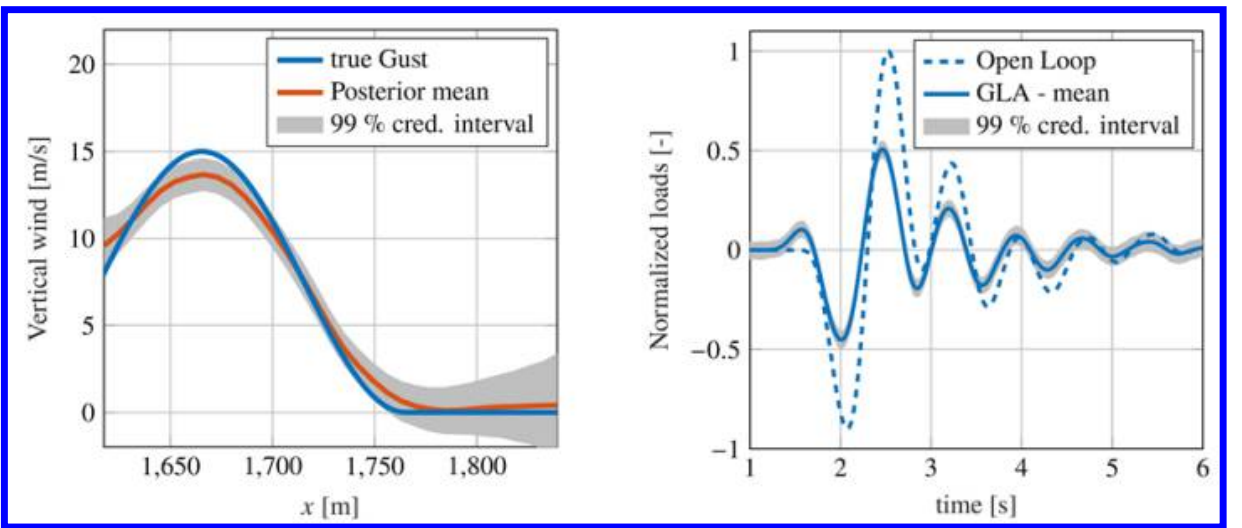

Fig. 17: Uncertainty quantification for a feed forward gust load alleviation system [24]

\section{Preliminary results on overall aircraft level}

The outputs of disciplinary technologies previously discussed in the paper have been integrated into ADEMAO via layers two and three for more detailed refinement and multidisciplinary optimization of the reference aircraft. The outputs are considered in terms of data look-up tables, surrogate models, or tool exchange, which were directly integrated with the design framework. The final design of the short-range aircraft includes a clean sheet sizing, low/medium-fidelity MDO [5], and physics/based design optimization for refinement [6]. The final design assuming a 2050 EIS is shown in Fig. 18. The characteristics of this aircraft are compared to the ATR 72 in Table 1.

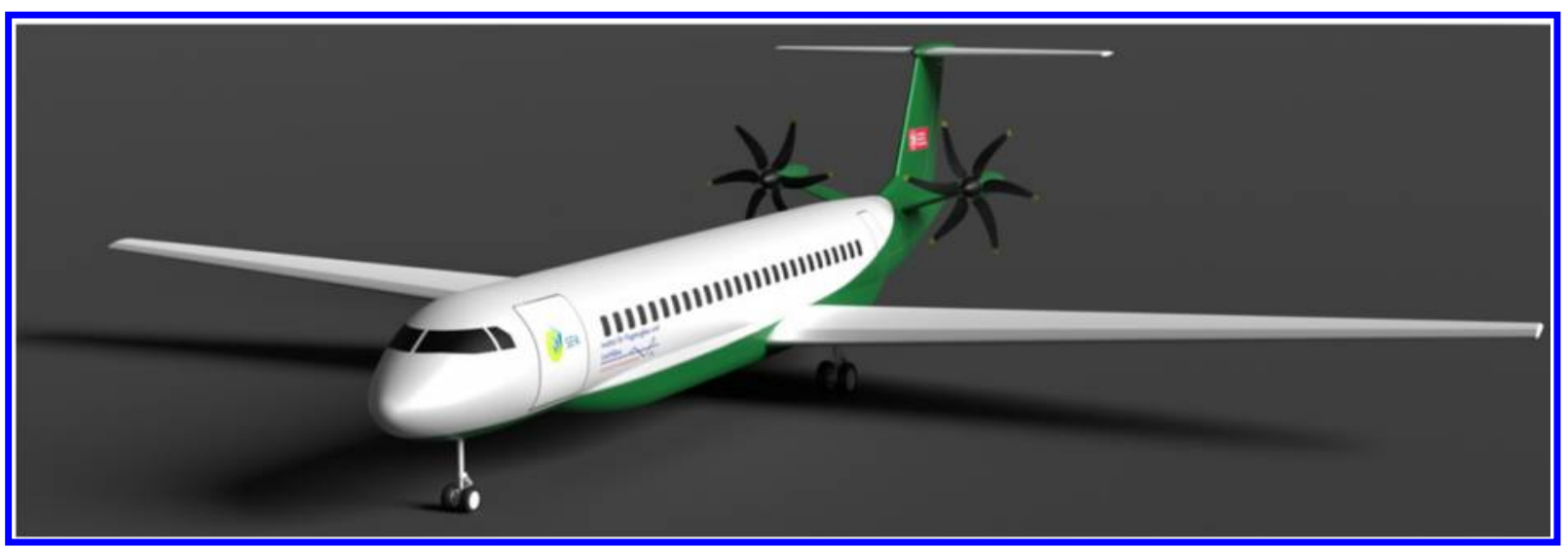

Figure 18: Final design of the $\mathrm{SE}^{2} \mathrm{~A}$ short range aircraft [5].

Table 1: Characteristics of the $\mathrm{SE}^{2} \mathrm{~A}$ short-range aircraft compared to the ATR-72.

\begin{tabular}{|l|c|c|}
\hline Parameter & SE$^{2}$ A Short Range Aircraft & ATR-72 \\
\hline MTOW [kg] & 34,441 & 23,000 \\
\hline OEW[kg] & 26,941 & 13,311 \\
\hline Battery Weight $[\mathrm{kg}]$ & 12,262 & - \\
\hline
\end{tabular}

While state-of-the-art battery technology does not allow full battery-electric aircraft for regional aviation, the operative emission capability in combination with net energy cost are strong leverage for future battery technologies with improved energy density. To investigate and assess the influence of new technologies mentioned in this paper on the realization of full battery electric regional aircraft, a series of sensitivity analyses have been performed on the short-range design of $\mathrm{SE}^{2} \mathrm{~A}$. The following technologies (ref. Chapter IV) were used: $50 \%$ drag reduction by HLFC and high-aspect ratio wings, limit load of 1.5 , high power electric motors $(99,5 \%$ eff.), cables $(99,5 \%$ eff.) and PMAD (99\% eff.) The results are shown in Fig. 19. From this figure, one can observe the significant influence of the new airframe technologies on realizing full electric aircraft. Without these technologies, the required battery 
weight severely increases the aircraft total weight, which is considered a showstopper in full battery-electric aircraft design. However, including and combining the technologies significantly reduces the total weight and allows the realization of full-electric aircraft with lower values of battery energy densities.
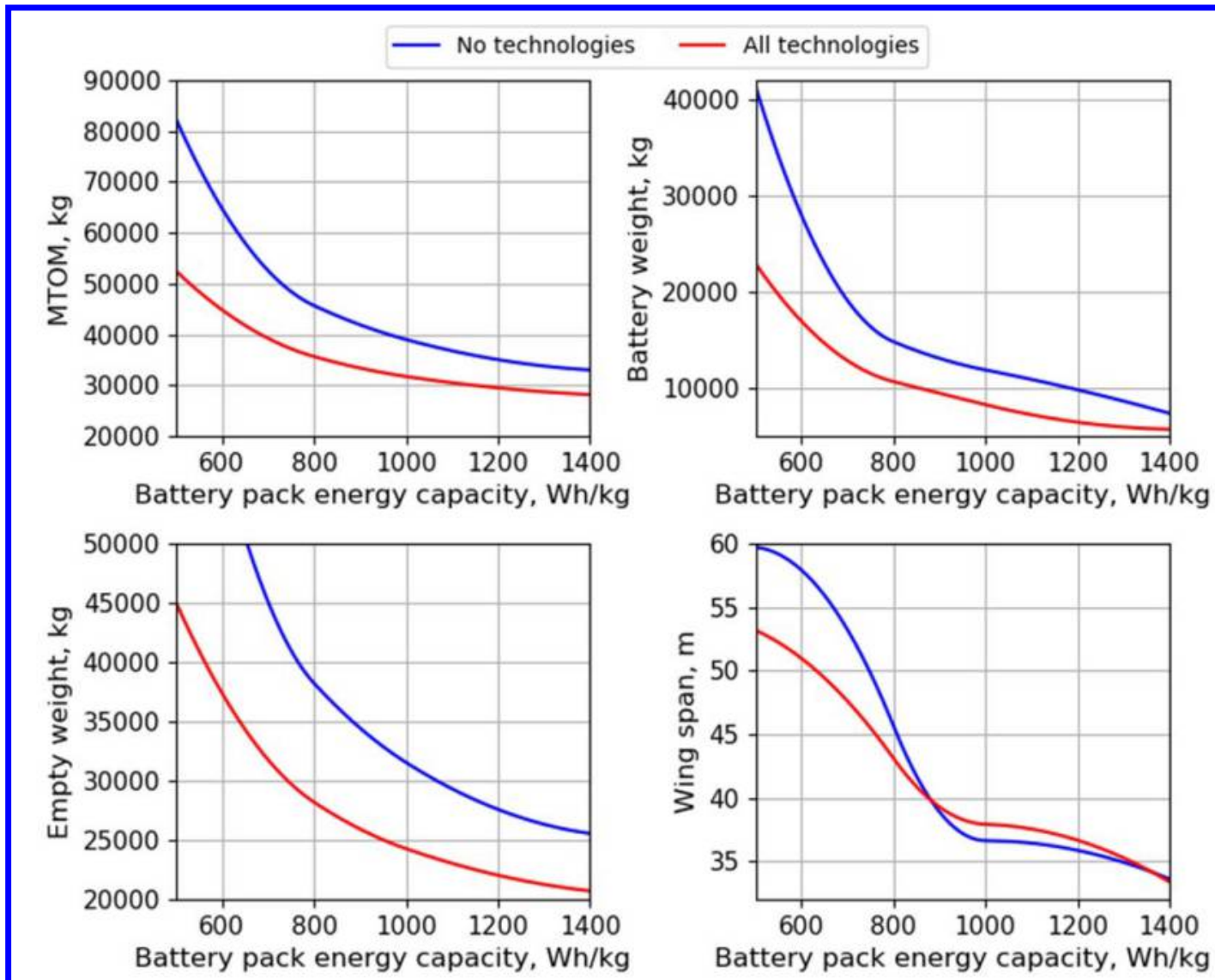

\section{Figure 19: Sensitivity analysis of short range full electric aircraft design with and without additional vehicle technologies (Cruise altitude $7300 \mathrm{~m}, M=0,42$ ).}

For the mid-range configuration following a similar approach, a drag reduction up to $50 \%$ and a structural mass reduction of $40 \%$ where assumed (EIS 2050), limit load of $2.0 \mathrm{~g}$ and UHBR fans with $25 \%$ SFC reduction. With regards to engine integration with BLI and according Power-Saving-Coefficients (PSC) the aft fuselage PSC can be predicted with low uncertainty in the range of $5-8 \%$ while the over-the-wing engine is affected by a more sensitive tradeoff between wing and engine aerodynamics which can also lead to a pylon mounted solution as the more robust result. The MDO study on both configurations of the mid-range aircraft shows that the forward-swept wing configuration is more efficient in terms of energy consumption [36]. Table 2 summarizes the characteristics of both forward- and backward-swept wing aircraft in direct comparison with to the Airbus A320. The preferred design is shown in Fig. 20.

These aircraft design data for the regional and mid-range reference aircraft demonstrate that the significance of technology performance indicators can be very well quantified using fully iterated aircraft designs. This allows the SE2A cluster to justify selection of specific research topics and to steer these thrusts into their most effective directions.

Table 2: Characteristics of the $\mathrm{SE}^{2} \mathrm{~A}$ mid-range aircraft compared to the A320-200.

\begin{tabular}{|l|c|c|c|}
\hline Parameter & Forward Swept wing & Backward Swept wing & A320-200 \\
\hline MTOW [kg] & 69000 & 72642 & 78000 \\
\hline OEW [kg] & 40496 & 42793 & 42600 \\
\hline L/D average cruise & 22.0 & 19.70 & 15.9 \\
\hline Mission fuel with contingency $[\mathrm{kg}]$ & 8840 & 10252 & 15500 \\
\hline
\end{tabular}




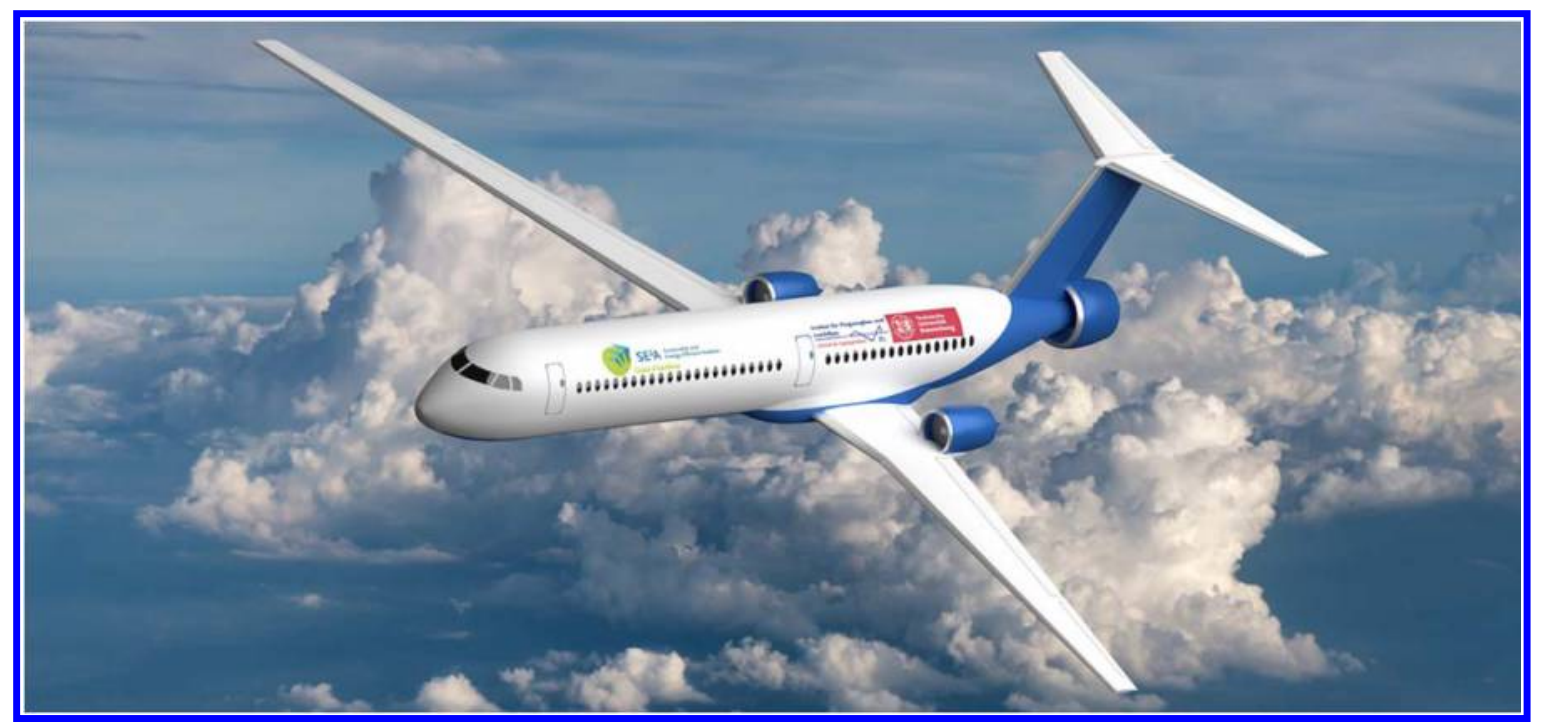

Fig. 20: Final design of the SE2A mid-range aircraft

\section{Summary \& Conclusion}

The Cluster of Excellence $\mathrm{SE}^{2} \mathrm{~A}$ has established a research structure which combines the competences and strengths of the aeronautical research with economic research as well a process and industrial engineering of Technische Universität Braunschweig, Leibniz University Hannover, German Aerospace Center, the National Metrology Institute of Germany and the Braunschweig University of Art (HBK). The overall mission is to develop the scientific and technological foundations for a sustainable future global air transport system and its required technologies. To fulfill its mission, the Cluster is organized in Integrated Cluster Areas which all have integrating projects coping with the assessment of underlying technologies and their performance indicators on system levels. For ICA B (Flight physics and vehicle systems) this is provided within "Design Methodology and Assessment of Aircraft". First conceptual studies on the impact of integration of novel technologies (Laminarization, Load Control, Mass Reduction) on aircraft level have shown that this approach is able identify promising subsystems technologies and to quantify the potential on aircraft level. Prerequisite of such assessment is a sound research at the according subsystem level using high-fidelity methods which allow not only to describe the key parameters and improvement of subsystems, but also to correctly predict the influence of sensitive parameters on a system level. First results of overall aircraft concept and optimization studies underline the importance of new technologies to reduce the required aircraft mission energy by extensively reducing the aircraft drag, loads and thus structural mass. Only the combination of such technologies with future energy and propulsion systems onboard of an aircraft will pave the way to sustainable aviation of the future.

\section{Acknowledgments}

We would like to acknowledge the funding by the Deutsche Forschungsgemeinschaft (DFG, German Research Foundation) under Germany's Excellence Strategy-EXC 2163/1-Sustainable and Energy Efficient AviationProject-ID 390881007. Furthermore, we acknowledge support by the Open Access Publication Funds of the Technische Universität Braunschweig.

\section{References}

[1] Schmidt, P., Weindorf, W., Roth, A. and Batteiger, V., "Power-to-Liquids - Potentials and Perspectives for the Future Supply of Renewable Aviation Fuel“, Umweltbundesamt, September 2016, https://www.umweltbundesamt.de/en/publikationen/power-to-liquids-potentials-perspectives-for-the

-[2] Radespiel, R., Heinze, W., "SFB 880: fundamentals of high lift for future commercial aircraft" CEAS Aeronautical Journal 5, pages 239-251 (2014), 2014, http://dx.doi.org/10.1007/s13272-014-0103-6 
-[3] Rang, J., Heinze, W., "An Optimal Configuration of an Aircraft with High Lift Configuration Using Surrogate Models and Optimization Under Uncertainties", in Advances in Structural and Multidisciplinary Optimization, Springer International Publishing AG, 2018, http:// http://dx.doi.org/10.1007/978-3-319-67988-4_29.

-[4] Giesecke, Lehmler, M., Friedrichs, J., Blinstrub, J., Bertsch, L., Heinze, W., "Evaluation of ultra-high bypass ratio engines for an over-wing aircraft configuration". Journal of the Global Power and Propulsion Society, 2: 493-515, 2018. https://doi.org/10.22261/JGPPS.8SHP7K

[5] Karpuk, S.; Elham, A., "Influence of Novel Airframe Technologies on the Feasibility of Fully-Electric Regional Aviation". Aerospace 2021, 8, 163. https://doi.org/10.3390/aerospace8060163

[6] Mosca, V., Karpuk, S., Sudhi, A., Badrya, C., Elham, A., "Multidisciplinary design optimisation of a fully electric regional aircraft wing with active flow control technology". The Aeronautical Journal, 1-25, 2021. doi:10.1017/aer.2021.101

[7] Karpuk, S., Liu, Y., Elham, A., "Multi-Fidelity Design Optimization of a Long-Range Blended Wing Body Aircraft with New Airframe Technologies", Aerospace 2020,7(7), 87, https://doi.org/10.3390/aerospace7070087

[8] Gunn, E. J., and Hall, C. A., “Aerodynamics of Boundary Layer Ingesting Fans”, GT2014-26142, ASME Turbo Expo, June 16-20, Düsseldorf, Germany, 2014.

[9] Budziszewski, N., and Friedrichs, J., "Modelling of a Boundary Layer Ingesting Propulsor”, Energies, Vol. 11, No. 4, 2018, doi:10.3390/en11040708

[10] Giesecke, D.; Friedrichs, J., “Aerodynamic Comparison between Circumferential and Wing-Embedded Inlet Distortion for an Ultra-High Bypass Ratio Fan Stage”. ASME GT2019-90425. https://doi.org/10.1115/GT2019-90425.

[11] Beck, N., Landa, T., Seitz, A., Boermans, L., Liu, Y., Radespiel, R., Drag Reduction by Laminar Flow Control, Energies, vol. 11, no. 1, p. 252, 2018.

[12] Bongers, J. Implementation of a new Transition Prediction Method in XFOIL. Master Thesis; Delft 848 University of Technology, Delft, 2006.

[13] van Ingen, J.L., 50 years $\mathrm{e}^{\wedge}$ N. Historical Review 1956-2006 of Work at Delft Aerospace Low Speed Laboratory including a New Version of the Method, 2006, https://surfdrive.surf.nl/files/index.php/s/JNqoleDxl1ZREMP.

[14] Schrauf, G., “LILO 2.1 User’s Guide and Tutorial,” GSSC TR 6, Bremen, Germany, Vol. 6, 2006.

[15] Sudhi, A., Elham, A., and Badrya, C., Coupled Boundary-Layer Suction and Airfoil Shape Optimization for Hybrid Laminar Flow Control, AIAA Journal. Sep. 2021. DOI: 10.2514/1.J060480

[16] Sudhi, A., Radespiel, R., and Badrya, C., Design of Transonic Swept Wing for HLFC Application, AIAA AVIATION 2021 Forum. DOI: 10.2514/6.2021-2606.

[17] Prasannakumar, A., Wolf, J., Radespiel, R., Boermans, L., Hühne, C., Badrya, C.; CEAS Aeronautical Journal, under review, 2021

[18] Grappadelli, M. C., Sattler, S., Scholz, P., Radespiel, R., Badrya, C.: Experimental investigations of boundary layer transition on a flat plate with suction, AIAA-2021-1452, https://doi.org/10.2514/6.2021-1452

[19] Bramsiepe, K., Gröhlich, M., Dähne, S., Hahn, D., "Structural Concepts for Passive Load Alleviation”, AIAA SciTech 2022.

[20] Haupt, M., Nies, R., Unger, R., and Horst, P., "Computational aero-structural coupling for hypersonic applications," 9th AIAA/ASME Joint Thermophysics and Heat Transfer Conference, 2006, p. 3252.

[21] Hahn, D., Haupt, M., and Heimbs, S., "Passive Load Alleviation by Nonlinear Stiffness of Airfoil Structures," AIAA SciTech Forum 2022, 2022.

-[22] Xu, J.; Kroo, I., “Aircraft Design with Maneuver and Gust Load Alleviation,” Proceedings of 29th AIAA Applied Aerodynamics Conference 2011, Honolulu, Hawaii, USA, June 27-30, 2011. https://doi.org/10.2514/6.2011-3180.

-[23] Zhao, Y.; Yue, C.; Hu, H., “Gust Load Alleviation on a Large Transport Airplane,” Journal of Aircraft, Vol. 53, 2016, pp. 1-15. https://doi.org/10.2514/6.2011-3180

[24] Bauknecht, A.; Beyer, Y.; Schultz, J.; Asaro, S.; Khalil, K.; Römer, U.; Steen, M.; Cavaliere, D.; Fezans, N., "Novel Concepts for Active Load Alleviation," Proceedings of the 2022 AIAA SciTech Forum and Exposition, San Diego, CA, USA, January 3-7, 2022.

[25] Leishman, J.; Nguyen, K., "State-space representation of unsteady airfoil behavior," AIAA Journal, Vol. 28, No. 5, 1990, pp. 836-844. 
-[26] Leishman, J.; Beddoes, T., “A Semi-Empirical model for dynamic stall,” Journal of the American Helicopter Society, Vol. 34, No. 3, 1989, pp. 3-17.

[27] Khalil, K.; Asaro, S.; Bauknecht, A., “Active flow control devices for wing load alleviation,” Journal of Aircraft, available online, 2021, pp. 1-17. https://doi.org/10.2514/1.C036426

[28] Asaro, S.; Lüer, L.; Bauknecht, A., "Experimental Characterization of a Dual-Slot Coandă Actuator for Load Reduction," Proceedings of the 2022 AIAA SciTech Forum and Exposition, San Diego, CA, USA, January 3-7, 2022.

[29] Traub, H., Wolff, J., Jose, S., Lobitz, L., Schollerer, M., Hühne, C., Concept and design of extended hybrid laminar flow control suction panels, DOI 10.21203/rs.3.rs-924184/v1, 2021.

[30] Pohya, A., Selected Current Challenges in the Development of Hybrid Laminar Flow Control on Transport Aircraft, Deutscher Luft- und Raumfahrtkongress, 2019.

-[31] Krishnan, K.S.G; Bertram, O; Seibel, O.: Review of hybrid laminar flow control systems. Progress in Aerospace Sciences 93 (2017), S. 24-52.

[32] Horn, M., Seitz, A., Schneider, M., Novel tailored skin single duct concept for HLFC fin application, DOI: DOI: 10.13009/EUCASS2017-44, 2017.

[33] Schrauf, G., Horstmann, K.H., Simplified Hybrid Laminar Flow Control, European Congress on Computational Methods in Applied Sciences and Engineering, Jyväskylä, 2004.

[34] Braunschweig, Technische Universität. "SE²A - Sustainable and Energy-Efficient Aviation.”, 2021. URL https://www.tu-braunschweig.de/se2a.

-[35] Abueidda, D; Elhebeary, M; Shiang, C.-S; Pang, S; Abu Al-Rub, R; Jasiuk, I.: Mechanical properties of 3D printed polymeric Gyroid cellular structures: Experimental and finite element study. Materials \& Design 165 (2019), p. 107597.

[36] Karpuk, S., Elham, A., Conceptual Design Trade Study for an Energy-Efficient Mid-Range Aircraft with Novel Technologies, AIAA SciTech 2021, 11-15 January 2021. 